TRANSACTIONS OF THE

AMERICAN MATHEMATICAL SOCIETY

Volume 355, Number 5, Pages 1819-1841

S 0002-9947(03)03238-0

Article electronically published on January 8, 2003

\title{
HUMBERT SURFACES AND THE KUMMER PLANE
}

\author{
CHRISTINA BIRKENHAKE AND HANNES WILHELM
}

\begin{abstract}
A Humbert surface is a hypersurface of the moduli space $\mathcal{A}_{2}$ of principally polarized abelian surfaces defined by an equation of the form $a z_{1}+$ $b z_{2}+c z_{3}+d\left(z_{2}^{2}-z_{1} z_{3}\right)+e=0$ with integers $a, \ldots, e$. We give geometric characterizations of such Humbert surfaces in terms of the presence of certain curves on the associated Kummer plane. Intriguingly this shows that a certain plane configuration of lines and curves already carries all information about principally polarized abelian surfaces admitting a symmetric endomorphism with given discriminant.
\end{abstract}

Let $\left(X, L_{0}\right)$ be a principally polarized abelian surface over the field of complex numbers. Its endomorphism $\operatorname{ring} \operatorname{End}(X)$ is either $\mathbb{Z}$, an order in a real quadratic number field, an order in an indefinite quaternion algebra over $\mathbb{Q}$, an order in a quartic CM field or, if $X$ is isogenous to a product of elliptic curves, $\operatorname{End}(X)$ is either $\mathbb{Z} \times \mathbb{Z}$, an order in $M_{2}(F)$ with $F=\mathbb{Q}$ or an imaginary quadratic field. Of course, in the general case $\operatorname{End}(X)=\mathbb{Z}$. In any other case $X$ admits a further endomorphism which is symmetric with respect to the Rosati involution defined by $L_{0}$. The Humbert surface $\mathcal{H}_{\Delta}$ with invariant $\Delta$ is the space of principally polarized abelian surfaces $\left(X, L_{0}\right)$ admitting a symmetric endomorphism with discriminant $\Delta$. It turns out that $\Delta$ is a positive integer $\equiv 0$ or $1 \bmod 4$.

Recall that the moduli space $\mathcal{A}_{2}$ of principally polarized abelian surfaces is the quotient of the Siegel upper half space $\mathfrak{H}_{2}$ of symmetric complex $2 \times 2$ matices with positive definite imaginary part by the action of the symplectic group $\mathrm{Sp}_{4}(\mathbb{Z})$. In these terms Humbert surfaces can be defined by equations. In fact, $\mathcal{H}_{\Delta}$ is the image in $\mathcal{A}_{2}$ of the zero locus in $\mathfrak{H}_{2}$ of any equation of the form

$$
a z_{1}+b z_{2}+c z_{3}+d\left(z_{2}^{2}-z_{1} z_{3}\right)+e=0
$$

with integers $a, b, c, d$ and $e$ satisfying $\Delta=b^{2}-4 a c-4 d e$ and $Z=\left(\begin{array}{c}z_{1} \\ z_{2} \\ z_{3}\end{array}\right) \in \mathfrak{H}_{2}$. Note that the equation is not uniquely determined because of the action of the symplectic group. The name Humbert surface is due to George Humbert who studied the zero loci (囝) in $[\underline{\mathrm{H}}]$.

In his paper $[\mathrm{H}]$, Humbert discovered a marvelous relationship between principally polarized abelian surfaces and a certain plane configuration of six lines. To be more precise, let $\left(X, L_{0}\right)$ be a principally polarized abelian surface and $K_{X}$ its Kummer surface in $\mathbb{P}_{3}$, i.e., the image of the morphism $\varphi$ defined by the linear system $\left|L_{0}^{2}\right|$. The 2-torsion points of $X$ map one-to-one to the 16 double points of $K_{X}$. Due to the $16_{6}$ configuration of $K_{X}$ there are six hyperplanes $P_{1}, \ldots, P_{6}$

Received by the editors February 11, 2002.

2000 Mathematics Subject Classification. Primary 14K10; Secondary 14H50.

Supported by DFG-contracts Bi 448/4-1 and $\mathrm{Hu} 337 / 5-1$. 
containing the double point $\varphi(0)$ and touching $K_{X}$ along a double conic. The linear projection with center $\varphi(0)$ maps these six hyperplanes onto six lines $l_{1}, \ldots, l_{6}$ in $\mathbb{P}_{2}$. We call the configuration $\left(\mathbb{P}_{2}, l_{1}, \ldots, l_{6}\right)$ the Kummer plane associated to $\left(X, L_{0}\right)$. The Kummer plane inherits essential information of the principally polaried abelian surface. For example for $\Delta=5$ Humbert showed under some generality assumptions:

Suppose $\left(X, L_{0}\right)$ is an irreducible principally polarized abelian surface. Then $\left(X, L_{0}\right) \in \mathcal{H}_{5}$ if and only if the associated Kummer plane $\left(\mathbb{P}_{2}, l_{1}, \ldots, l_{6}\right)$ admits a smooth conic passing through five of the 15 points $\left\{l_{i} \cap l_{j}\right\}$ and touching one singular line.

Humbert proved similar results for the invariants $\Delta=4,8,9$ and 12 as well as some families of invariants.

In this paper we present a systematic approach to Humbert's ideas. In fact, we give new proofs for Humbert's results. This enables us not only to get rid of his generality assumptions but also to extend his work to other invariants. For example we show

Theorem 7.1. Suppose $\Delta=8 d^{2}+9-2 k$ with $d \geq 1$ and $k \in\{4,6,8,10,12\}$. If $\left(X, L_{0}\right) \in \mathcal{H}_{\Delta}$ is an irreducible principally polarized abelian surface, then the associated Kummer plane $\left(\mathbb{P}_{2}, l_{1}, \ldots, l_{6}\right)$ admits a rational curve $C^{\prime}$ of degree $2 d$ passing smoothly through exactly $k-1$ points of $\left\{l_{i} \cap l_{j}\right\}$ and touching the singular lines $l_{i}$ in the remaining intersection points with even multiplicity. Conversely, if $\left(\mathbb{P}_{2}, l_{1}, \ldots, l_{6}\right)$ admits such a curve, then $\left(X, L_{0}\right) \in \mathcal{H}_{\Delta^{\prime}}$ with $\Delta^{\prime} \leq \Delta$.

For small invariants $\Delta$ the inequality $\Delta^{\prime} \leq \Delta$ in the last sentence can be improved (see Corollaries 7.2 and 7.3). Since Theorems 7.1 to 7.6 cover all possible invariants $\Delta$ we present geometric characterizations of all Humbert surfaces.

The paper is structured as follows: in Sections 1 and 2 we collect some wellknown facts about principally polarized abelian surfaces and the Kummer surface. In Section 3 we compute the genus of curves on the Kummer surface and derive some consequences. In Section 4 the relation between Humbert surfaces, singular relations such as (因) and symmetric endomorphisms is developed. Moreover, we prove some first observations about Humbert surfaces. In Section 5 we translate the definition of Humbert surfaces into terms of line bundles and in Section 6 we study curves on the Kummer plane. Finally, Section 7 presents the results.

Part of this paper was the doctoral thesis of the second author, written under the supervision of the first author.

\section{Preliminaries}

In this section we collect some well-known results on abelian surfaces and Kummer surfaces and introduce the notation. For proofs and more details we refer to CAV.

An abelian surface $X$ is an algebraic complex torus $V / \Lambda$ of dimension 2 with a $\mathbb{C}$-vector space $V \simeq \mathbb{C}^{2}$ and a lattice $\Lambda \simeq \mathbb{Z}^{4}$ in $V$. A polarization on $X$ is a positive definite Hermitian form $H: V \times V \longrightarrow \mathbb{C}$ whose imaginary part takes integer values on $\Lambda$. The polarization is called principal, if $\operatorname{Im} H$ is unimodular. The pair $(X, H)$ is called polarized abelian surface. If $L$ is a line bundle on $X$ with first Chern class $H$ we also call $(X, L)$ polarized abelian surface. Consider the Siegel upper halfspace $\mathfrak{H}_{2}=\left\{Z \in M_{2}(\mathbb{C}) \mid{ }^{t} Z=Z\right.$ and $\left.\operatorname{Im} Z>0\right\}$. An element $Z=\left(\begin{array}{ll}z_{1} & z_{2} \\ z_{2} & z_{3}\end{array}\right) \in \mathfrak{H}_{2}$ 
determines a principally polarized abelian surface $\left(X_{Z}, H_{0}\right)$, where

$$
X_{Z}=\mathbb{C}^{2} /\left(Z, \mathbf{1}_{2}\right) \mathbb{Z}^{4}
$$

and $H_{0}$ is the Hermitian form

$$
\mathbb{C}^{2} \times \mathbb{C}^{2} \longrightarrow \mathbb{C}, \quad H_{0}(v, w)={ }^{t} v(\operatorname{Im} Z)^{-1} \bar{w} .
$$

Denote $\Lambda_{0}=\left(Z, \mathbf{1}_{2}\right) \mathbb{Z}^{4}$. The four columns $\left(\begin{array}{l}z_{1} \\ z_{2}\end{array}\right),\left(\begin{array}{l}z_{2} \\ z_{3}\end{array}\right),\left(\begin{array}{l}1 \\ 0\end{array}\right),\left(\begin{array}{l}0 \\ 1\end{array}\right)$ of $\left(Z, \mathbf{1}_{2}\right)$ are a basis of the lattice $\Lambda_{0}$. With respect to this basis the alternating form $E_{0}=$ $\operatorname{Im} H_{0} \mid \Lambda_{0}$ is given by the matrix $\left(\begin{array}{cc}0 & \mathbf{1}_{2} \\ -\mathbf{1}_{2} & 0\end{array}\right)$. The symplectic group $\operatorname{Sp}_{4}(\mathbb{Z})$ acts on $\mathfrak{H}_{g}$ by $Z \mapsto M(Z):=(\alpha Z+\beta)(\gamma Z+\delta)^{-1}$ for $M=\left(\begin{array}{cc}\alpha & \beta \\ \gamma & \delta\end{array}\right) \in \operatorname{Sp}_{4}(\mathbb{Z})$. The quotient $\mathcal{A}_{g}:=$ $\mathfrak{H}_{g} / \mathrm{Sp}_{2 g}(\mathbb{Z})$ is a coarse moduli space for principally polarized abelian surfaces. In particular, every principally polarized abelian surface is of the form $\left(X_{Z}, H_{0}\right)$, and two such pairs $\left(X_{Z}, H_{0}\right)$ and $\left(X_{Z^{\prime}}, H_{Z^{\prime}}\right)$ are isomorphic if and only if $Z^{\prime}=M(Z)$ for some $M \in \mathrm{Sp}_{4}(\mathbb{Z})$.

Let $X=V / \Lambda$ be an abelian surface and $L$ a line bundle on $X$. The first Chern class $c_{1}(L)$, i.e., the image of $L$ in the Néron-Severi group $\mathrm{NS}(X)$, is a Hermitian form $H$ on $V$ with $\operatorname{Im} H(\Lambda, \Lambda) \subseteq \mathbb{Z}$. Two line bundles $L_{1}$ and $L_{2}$ are algebraically equivalent, denoted by $L_{1} \equiv L_{2}$, if their first Chern classes coincide. The type of a line bundle or of its first Chern class $H$, respectively, is the pair $\left(d_{1}, d_{2}\right)$ of elementary divisors of the alternating form $\operatorname{Im} \mid \Lambda$. Note that always $d_{1} \mid d_{2}$. For an ample line bundle $L$ of type $\left(d_{1}, d_{2}\right)$ the dimension of $H^{0}(L)$ is $h^{0}(L)=d_{1} d_{2}$.

For $n \in \mathbb{Z}$ denote by $n_{X}$ the multiplication by $n$ on $X$. A line bundle $L$ on $X$ is called symmetric if $(-1)_{X}^{*} L \simeq L$. If $L$ is symmetric, then $(-1)_{X}$ lifts to an involution $(-1)_{L}$ on $L$ which acts as the identity on the fibre $L(0)$ over 0 (see [CAV] Lemma 4.6.3). $(-1)_{L}$ induces an involution on $H^{0}(L)$ splitting it into eigenspaces

$$
H^{0}(L)=H^{0}(L)^{+} \oplus H^{0}(L)^{-} \text {. }
$$

Since $L$ is symmetric, $(-1)_{L}$ acts by multiplication with +1 or -1 on the fibre $L(x)$. Consider the sets

$$
X_{2}^{ \pm}(L):=\left\{x \in X_{2}\left|(-1)_{L}\right| L(x)= \pm 1\right\} .
$$

For the cardinalities of these sets we have

Proposition 1.1. Suppose $L$ is an ample symmetric line bundle of type $\left(d_{1}, d_{2}\right)$. Then

$$
\# X_{2}^{+}(L) \in \begin{cases}\{8,16\} & \text { if } d_{1} \text { is even, } \\ \{4,8,12\} & \text { if } d_{1} \text { is odd and } d_{2} \text { is even, } \\ \{6,10\} & \text { if } d_{2} \text { is odd, }\end{cases}
$$

and all cases occur.

Proof. This is a consequence of CAV 4.7.7 and 4.(14).

Lemma 1.2. For ample $L$ we have $h^{0}(L)^{ \pm}=\frac{1}{4}\left(\left(L^{2}\right)-\# X_{2}^{\mp}(L)\right)+2$.

For a proof see [B] Theorem 3.1 and the remark after Proposition 3.5

Let $\vartheta \in H^{0}(L)$ and $D=(\vartheta)$ be the corresponding effective divisor on $X$. A symmetric divisor $D$ is called even (respectively odd), if $\vartheta \in H^{0}(L)^{+}\left(H^{0}(L)^{-}\right.$ respectively), or equivalently if mult $_{0} D$ is even (odd respectively). Consider the set

$$
X_{2}^{ \pm}(D):=\left\{x \in X_{2} \mid(-1)^{\text {mult }_{x} D}= \pm 1\right\}
$$


of 2-torsion points where $D$ has even or odd respectively multiplicity. Clearly, if $D$ is even (respectively odd) $X_{2}^{-}(D)\left(X_{2}^{+}(D)\right.$ respectively) is contained in $D$. By [CAV] Proposition 4.7 .2 we have $X_{2}^{ \pm}(D)=X_{2}^{ \pm}(L)$, if $D$ is even, and $X_{2}^{ \pm}(D)=X_{2}^{\mp}(L)$, if $D$ is odd. In particular, the cardinality of $X_{2}^{ \pm}(D)$ is always even.

Now suppose $L_{0}$ is a symmetric line bundle on $X$ defining a principal polarization. Denote by $t_{x}: X \longrightarrow X$ the translation by a point $x \in X$. It is easy to see that $L=t_{x}^{*} L_{0}^{n}$ is symmetric if and only if $x \in X_{2 n}$. In particular, since $\# X_{2}=16$, there are exactly 16 symmetric line bundles $t_{x}^{*} L_{0}, x \in X_{2}$. For $x \in X_{2}$ the linear system $\left|t_{x}^{*} L_{0}\right|$ consists of one divisor $D$ such that $2 D \in\left|t_{x}^{*} L_{0}^{2}\right|=\left|L_{0}^{2}\right|$. Moreover, the line bundle $L_{0}^{2}$ is symmetric and $H^{0}\left(L_{0}^{2}\right)=H^{0}\left(L_{0}^{2}\right)^{+}$.

\section{The Kummer Surface And the $16_{6}$-Configuration}

Suppose $\left(X, L_{0}\right)$ is an irreducible principally polarized abelian surface. The line bundle $L_{0}^{2}$ defines a morphism $\varphi=\varphi_{L_{0}^{2}}: X \longrightarrow \mathbb{P}_{3}$. Since $L_{0}^{2}$ is symmetric and $H^{0}\left(L_{0}^{2}\right)=H^{0}\left(L_{0}^{2}\right)^{+}$, the morphism factorizes via the action of $(-1)_{X}$ on $X$ :

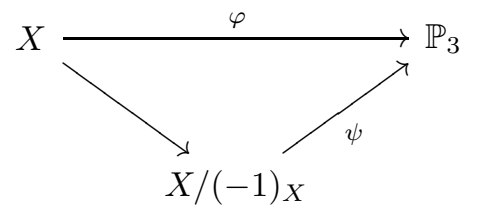

The projection $X \longrightarrow X /(-1)_{X}$ is a double covering ramified at the 162 -torsion points $X_{2}$. The morphism $\psi: X /(-1)_{X} \hookrightarrow \mathbb{P}_{3}$ is an embedding. Its image

$$
K_{X}:=\varphi(X)
$$

is called the Kummer surface associated with $X$ and $\varphi: X \longrightarrow K_{X}$ is called the Kummer map. The Kummer surface $K_{X}$ is a quartic surface in $\mathbb{P}_{3}$, smooth apart from 16 singular points. These are ordinary double points and the images of the 162 -torsion points. In the notation we do not distinguish between the 2-torsion points on $X$ and the corresponding nodes of $K_{X}$.

On $X$ the notion of curves and effective divisors coincide, $X$ being a surface.

\section{Proposition 2.1.}

a) If $D \in\left|t_{x}^{*} L_{0}^{n}\right|, \quad x \in X_{2 n}$, is a symmetric divisor, then $\varphi(D)=C$ is a curve of degree $2 n$ on $K_{X}$ and $2 C$ is a complete intersection of $K_{X}$ with a hypersurface $S \subset \mathbb{P}_{3}$ of degree $n$.

b) Every complete intersection $K_{X} \cap S$ with a hypersurface $S$ of degree $n$ is the image of an even symmetric effective divisor $D^{\prime} \in\left|L_{0}^{2 n}\right|$.

In other words, symmetric divisors $D \in\left|t_{x}^{*} L_{0}^{n}\right|$ correspond to hypersurfaces $S$ of degree $n$ touching the Kummer surface along the curve $2 \varphi(D)=\varphi_{*}(D)$. The hyperplanes touching $K_{X}$ in this way are called singular planes. There are exactly 16 singular planes $P_{x}, x \in X_{2}$, corresponding to the 16 symmetric divisors $D_{x}=$ $\left|t_{x}^{*} L_{0}\right|, x \in X_{2}$, by

$$
P_{x} \cap K_{X}=2 \varphi\left(D_{x}\right) .
$$

The curve $C_{x}:=\varphi\left(D_{x}\right)$ is a conic on $K_{X}$. In these terms the famous $16_{6}$ configuration of $K_{X}$ reads as follows:

Proposition 2.2. Any singular plane contains exactly 6 singular points and any singular point is contained in exactly 6 singular planes. 
Moreover $K_{X}$ satisfies:

Proposition 2.3. Any two singular points belong to the intersection of exactly two singular planes and any two singular planes have exactly two singular points in common.

\section{Curves on the Kummer Surface}

In this section we study arbitrary curves on the Kummer surface $K_{X}$ of a principally polarized abelian surface $\left(X, L_{0}\right)$. Suppose $D$ is a symmetric divisor on $X$ defining a line bundle $L=\mathcal{O}_{X}(D)$. Let the curve $C:=\varphi(D)$ be its image on $K_{X}$. The Kummer map restricts to a double covering $\varphi: D \longrightarrow C$ ramified at the 2 -torsion points on $D$. In particular, we have

Lemma 3.1. $\operatorname{deg} C=\left(D \cdot L_{0}\right)$ and $\operatorname{mult}_{\varphi(x)} C=\operatorname{mult}_{x} D$ for $x \in X_{2}$.

The curves $C$ and $D$ may be singular and reducible (in any case they are connected, since any two curves on an abelian surface intersect). We assume that $D$ admits at most ordinary singularities, i.e., no singular point of $D$ admits multiple tangent directions. Our next aim is to compute the geometric genus of $C$. For this we need some notation: Let $\sigma: \tilde{X} \longrightarrow X$ be the blow-up of $X$ at the singular points of $D$. Denote by $E_{x}$ the exceptional divisor over a 2-torsion point $x \in X_{2}$ (if $x$ is either not contained in $D$ or a smooth point of $D$ we set $E_{x}=\emptyset$ ) and by $F_{j}$ the exceptional divisors over the remaining singular points $P_{j}$ (including infinitely near points). Moreover, denote $r_{x}:=$ mult $_{x} D$ for $x \in X_{2}$, and denote by $r_{j}$ the multiplicity of the singular point $P_{j}$. Then the proper transform

$$
\tilde{D}=\sigma^{*} D-\sum_{x \in X_{2}} r_{x} E_{x}-\sum_{j} r_{j} F_{j}
$$

of $D$ is a smooth curve on $\tilde{X}$. The involution $(-1)_{X}$ lifts to an involution $(-1)_{\tilde{X}}$ on $\tilde{X}$, the curve $D$ being symmetric. Let $\tilde{K}:=\tilde{X} /(-1)_{\tilde{X}}$ and denote by $\tilde{\varphi}: \tilde{X} \longrightarrow \tilde{K}$ the natural projection. The birational map $\sigma$ descends to a birational map $\tau$ fitting into the commutative diagram

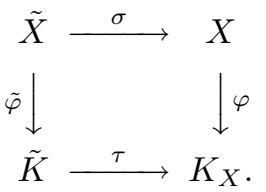

By construction $(-1)_{\tilde{X}}$ restricts to an involution $\iota$ on $\tilde{D}$. The quotient $\tilde{C}:=\tilde{D} / \iota=$ $\tilde{\varphi}(\tilde{D})$ is a smooth curve on $\tilde{K}$ and diagram (2) restricts to a commutative diagram

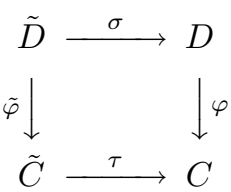

where the vertical maps are double coverings and the horizontal maps are birational. In particular, $\tilde{C}$ is the normalization of the curve $C$. 
Lemma 3.2. There is a commutative diagram with exact rows

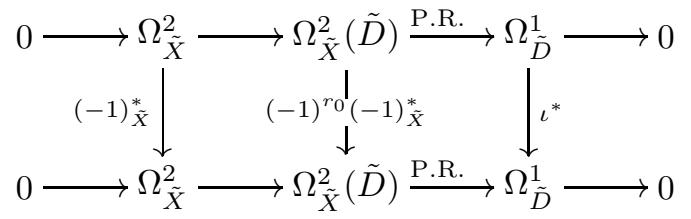

Here the rows are the Poincaré Residue sequence, P.R. is the Poincaré Residue map, $r_{0}=$ mult $_{0} D$ as above and $(-1)^{r_{0}}$ means multiplication with the number $(-1)^{r_{0}}$.

Proof. Let $z_{1}, z_{2}$ be local coordinates on $\tilde{X}$ and let $f\left(z_{1}, z_{2}\right)=0$ be a local equation for $\tilde{D}$. The map $\Omega_{\tilde{X}}^{2} \longrightarrow \Omega_{\tilde{X}}^{2}(\tilde{D})$ is given locally by multiplication with $f$. Now the first square commutes, since $(-1)_{\tilde{X}}^{*} f=(-1)^{r_{0}} f$. As for the second square, recall that for a local section $\theta$ of $\Omega_{\tilde{X}}^{2}(\tilde{D})$ the Poincaré Residue map is given by P.R. $(\theta)=\left.\frac{\theta d z_{1}}{\partial f / \partial z_{2}}\right|_{f=0}$. Elementary calculus gives $(-1)_{\tilde{X}}^{*} \frac{\partial f}{\partial z_{2}}=(-1)^{r_{0}+1} \frac{\partial f}{\partial z_{2}}$; hence

$$
\text { P.R. }\left((-1)_{\tilde{X}}^{*} \theta\right)=\left.\frac{(-1)_{\tilde{X}}^{*} \theta d z_{1}}{\partial f / \partial z_{2}}\right|_{f=0}=(-1)^{r_{0}} \iota^{*}\left(\left.\frac{\theta d z_{1}}{\partial f / \partial z_{2}}\right|_{f=0}\right)=(-1)^{r_{0}} \iota^{*} \text { P.R. }(\theta)
$$

implying that the second square is commutative.

This action of the involution on the Poincaré Residue sequence induces an action on its long cohomology sequence. Denoting by $H^{0}\left(\Omega_{\tilde{X}}^{2}(\tilde{D})\right)^{ \pm}$the \pm 1 eigenspaces with respect to $(-1)_{\tilde{X}}^{*}$ we obtain:

Proposition 3.3. The long exact cohomology sequence of the Poincaré Residue sequence induces the short exact sequence

$$
0 \longrightarrow H^{0}\left(\Omega_{\tilde{X}}^{2}\right) \longrightarrow H^{0}\left(\Omega_{\tilde{X}}^{2}(\tilde{D})\right)^{ \pm} \longrightarrow \tilde{\varphi}^{*} H^{0}\left(\Omega_{\tilde{C}}^{1}\right) \longrightarrow 0
$$

with "+", if $D$ is even, and "-", if $D$ is odd.

Proof. Writing $X=V / \Lambda$ as a complex torus, the vector space $V$ identifies with $H^{1}\left(\mathcal{O}_{X}\right)$. Under this identification the involution $(-1)_{X}^{*}$ on $H^{1}\left(\mathcal{O}_{X}\right)$ is just the analytic representation of $(-1)_{X}$, i.e., multiplication by -1 on $V$. On the other hand, Serre duality and the Hodge decomposition give

$$
H^{i}\left(\Omega_{\tilde{X}}^{2}\right) \simeq H^{2-i}\left(\mathcal{O}_{\tilde{X}}\right)^{*} \simeq H^{2-i}\left(\mathcal{O}_{X}\right)^{*} \simeq \bigwedge^{2-i} V^{*} .
$$

Hence $(-1)_{\tilde{X}}^{*}$ acts on $H^{0}\left(\Omega_{\tilde{X}}^{2}\right)$ by $\bigwedge^{2}(-\mathrm{id})=$ id and on $H^{1}\left(\Omega_{\tilde{X}}^{2}\right)$ by $\bigwedge^{1}(-\mathrm{id})=$ -id. It follows that the diagram of Lemma 3.2 induces the following commutative diagram in cohomology:

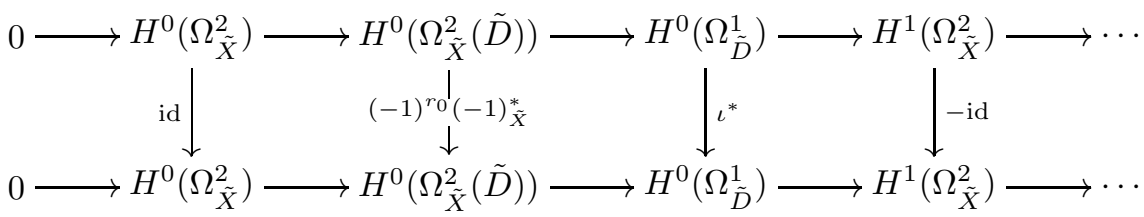

Noting that $\tilde{\varphi}^{*} H^{0}\left(\Omega_{\tilde{C}}^{1}\right)$ is the +1 -eigenspace of $H^{0}\left(\Omega_{\tilde{D}}^{1}\right)$ with respacet to $\iota^{*}$ the assertion follows from an immediate diagram chase.

Since $\tilde{\varphi}^{*}: H^{0}\left(\Omega_{\tilde{C}}^{1}\right) \longrightarrow H^{0}\left(\Omega_{\tilde{D}}^{1}\right)^{+}$is injective and since by definition the geometric genus $g_{C}$ of $C$ is the genus of its normalization $\tilde{C}$ we get as a consequence: 
Corollary 3.4. $g_{C}=h^{0}\left(\Omega_{\tilde{X}}(\tilde{D})\right)^{ \pm}-1$ with "+" if $D$ is even and "-" if $D$ is odd.

In order to compute $g_{C}$ explicitly denote by $\omega_{\tilde{X}}, \omega_{\tilde{D}}$, and $\omega_{\tilde{C}}$ the canonical sheafs. Then the Poincare Residue sequence may be considered as the natural restriction sequence $0 \longrightarrow \omega_{\tilde{X}} \longrightarrow \omega_{\tilde{X}}(\tilde{D}) \longrightarrow \omega_{\tilde{D}} \longrightarrow 0$. Denote by $\left|\omega_{\tilde{X}}(\tilde{D})\right|^{ \pm}$the sublinear system $H^{0}\left(\omega_{\tilde{X}}(\tilde{D})\right)^{ \pm} / \mathbb{C}$ of $\left|\omega_{\tilde{X}}(\tilde{D})\right|$. With this notation Proposition 3.3 states that restriction to $\tilde{D}$ induces an isomorphism $\operatorname{res}_{\tilde{D}}:\left|\omega_{\tilde{X}}(\tilde{D})\right|^{ \pm} \longrightarrow \tilde{\varphi}^{*} H^{0}\left(\omega_{\tilde{C}}\right)$. Consequently,

$$
\left(D^{\prime} \cdot \tilde{D}\right)=\operatorname{deg} D^{\prime} \mid \tilde{D}=\operatorname{deg} \tilde{\varphi}^{*} \omega_{\tilde{C}}=2\left(2 g_{C}-2\right)
$$

for any $D^{\prime} \in\left|\omega_{\tilde{X}}(\tilde{D})\right|^{ \pm}$. Writing $r_{x}=2 \mu_{x}+1$ for $x \in X_{2}^{-}(D)$ and $r_{x}=2 \mu_{x}$ for $x \in X_{2}^{+}(D)$, this gives

Proposition 3.5. The geometric genus $g_{C}$ of the curve $C=\varphi(D)$ is

$$
\frac{1}{4}\left(\left(L^{2}\right)-\# X_{2}^{-}(D)\right)+1-\sum_{x \in X_{2}^{-}(D)} \mu_{x}\left(\mu_{x}+1\right)-\sum_{x \in X_{2}^{+}(D)} \mu_{x}^{2}-\frac{1}{4} \sum_{j} r_{j}\left(r_{j}-1\right) .
$$

Note that $\frac{1}{4} \sum_{j} r_{j}\left(r_{j}-1\right)$ is an integer, since the singular points $P_{j}$ of $D$ occur in pairs, $D$ being symmetric.

Proof. Recall that $\omega_{\tilde{X}}=\mathcal{O}_{\tilde{X}}(E)$, where $E$ is the sum of all the exceptional divisors $E_{x}$ and $F_{j}$ without multiplicities. Hence we have by equation (1),$\omega_{\tilde{X}}(\tilde{D})=$ $\sigma^{*} L\left(-\sum_{x \in X_{2}}\left(r_{x}-1\right) E_{x}-\sum_{j}\left(r_{j}-1\right) F_{j}\right)$. So $\sigma$ induces an isomorphism $H^{0}\left(\omega_{\tilde{X}}(\tilde{D})\right)$ $\simeq H^{0}\left(L \otimes \bigotimes_{x \in X_{2}} \mathcal{I}_{x}^{r_{x}-1} \otimes \bigotimes_{j} \mathcal{I}_{P_{j}}^{r_{j}-1}\right)$ where $\mathcal{I}_{p}$ denotes the ideal sheaf of a point $p \in X$. Note that for any two symmetric sections $\vartheta_{1}, \vartheta_{2} \in H^{0}(L)$ of the same parity, the multiplicities at the 2 -torsion points are congruent modulo 2 , i.e., $(-1)^{\text {mult }_{x} \vartheta_{1}}=$ $(-1)^{\text {mult }_{x} \vartheta_{2}}$ for $x \in X_{2}$. Hence $H^{0}\left(L \otimes \bigotimes_{x \in X_{2}} \mathcal{I}_{x}^{r_{x}-1} \otimes \bigotimes_{j} \mathcal{I}_{P_{j}}^{r_{j}-1}\right)^{ \pm}=H^{0}(L \otimes$ $\left.\bigotimes_{x \in X_{2}} \mathcal{I}_{x}^{r_{x}} \otimes \bigotimes_{j} \mathcal{I}_{P_{j}}^{r_{j}-1}\right)^{ \pm}$and thus $\left|\omega_{\tilde{X}}(\tilde{D})\right|^{ \pm}$is actually a sublinear system of $\left|\sigma^{*} L\left(-\sum_{x \in X_{2}} r_{x} E_{x}-\sum_{j}\left(r_{j}-1\right) F_{j}\right)\right|$. So for $D^{\prime} \in\left|\omega_{\tilde{X}}(\tilde{D})\right|^{ \pm}$we have

$$
\begin{aligned}
& \left(D^{\prime} \cdot \tilde{D}\right) \\
& =\left(\sigma^{*} L\left(-\sum_{x \in X_{2}} r_{x} E_{x}-\sum_{j}\left(r_{j}-1\right) F_{j}\right)\right) \cdot\left(\sigma^{*} L\left(-\sum_{x \in X_{2}} r_{x} E_{x}-\sum_{j} r_{j} F_{j}\right)\right) \\
& =\left(\sigma^{*} L^{2}\right)-\sum_{x \in X_{2}} r_{x}^{2}-\sum_{j} r_{j}\left(r_{j}-1\right) \\
& =\left(L^{2}\right)-4 \sum_{x \in X_{2}^{-}(D)} \mu_{x}\left(\mu_{x}+1\right)-\# X_{2}^{-}(D)-4 \sum_{x \in X_{2}^{+}(D)} \mu_{x}^{2}-\sum_{j} r_{j}\left(r_{j}-1\right) .
\end{aligned}
$$

With equation (4) this implies the assertion.

Note that if $D$ is smooth this gives a new proof of Lemma 1.2. In fact, by Corollary 3.4 and Proposition 3.5 we get for smooth divisors $D: h^{0}(L)^{ \pm}=g_{C}+1=$ $\frac{1}{4}\left(\left(L^{2}\right)-\# X_{2}^{-}(L)^{\mp}\right)+2$, since $X_{2}^{-}(D)=X_{2}^{\mp}(L)$, with "-", if $D$ is even, and "+", if $D$ is odd. On the other hand, rewriting Proposition 3.5 by means of Lemma 1.2 we get

$$
g_{C}=h^{0}(L)^{ \pm}-1-\sum_{x \in X_{2}^{-}(D)} \mu_{x}\left(\mu_{x}+1\right)-\sum_{x \in X_{2}^{+}(D)} \mu_{x}^{2}-\frac{1}{4} \sum_{j} r_{j}\left(r_{j}-1\right)
$$

with "+", if $D$ is even, and "-", if $D$ is odd. 


\section{Lemma 3.6.}

$$
\begin{aligned}
h^{0}\left(L \otimes \mathcal{I}_{0}^{2 d}\right)^{+} & \geq h^{0}(L)^{+}-d^{2}, \\
h^{0}\left(L \otimes \mathcal{I}_{0}^{2 d+1}\right)^{-} & \geq h^{0}(L)^{-}-d(d+1) .
\end{aligned}
$$

Proof. Recall that $H^{0}\left(L \otimes \mathcal{I}_{0}^{2 d}\right)^{+}$is the subvector space of even theta functions for $L$ with mult $_{0} \vartheta \geq 2 d$ (and similarly for $H^{0}\left(L \otimes \mathcal{I}_{0}^{2 d+1}\right)^{-}$). For $\vartheta \in H^{0}(L)$ the multiplicity mult $\mathrm{t}_{0} \vartheta$ is the subdegree of the Taylor expansion of $\vartheta$ around 0 . If $\vartheta$ is even (or odd respectively), then its Taylor expansion involves only summands of even (or odd respectively) degree. Since the vector space of homogeneous polynomials of degree $\nu$ in 2 variables is of dimension $\nu+1$, the condition mult $_{0} \vartheta \geq 2 d$ (or $2 d+1$ respectively) imposes at most

$$
\sum_{\substack{\nu=0 \\ \nu \text { even }}}^{2 d-1}(\nu+1)=\sum_{\nu=1}^{d}(2 \nu-1)=d^{2}
$$

(or $\sum_{\substack{\nu=1 \\ \nu \text { odd }}}^{2 d}(\nu+1)=\sum_{\nu=1}^{d} 2 \nu=d(d+1)$ respectively) conditions on $H^{0}(L)^{ \pm}$. This implies the assertion.

Corollary 3.7. Let $L$ be a symmetric line bundle on $X$.

a) If $h^{0}(L)^{+}=d^{2}+g+1$, then the linear system $\left|L \otimes \mathcal{I}_{0}^{2 d}\right|^{+}$is of dimension $\geq g$ and its general member is smooth on $X-\{0\}$ and maps to curve $C=\varphi(D)$ of genus $g$ on $K$.

b) If $h^{0}(L)^{-}=d(d+1)+g+1$, then the linear system $\left|L \otimes \mathcal{I}_{0}^{2 d+1}\right|^{-}$is of dimension $\geq g$ and its general member is smooth on $X-\{0\}$ and maps to curve $C=\varphi(D)$ of genus $g$ on $K$.

Proof. a) By Lemma $3.6 \operatorname{dim}\left|L \otimes \mathcal{I}_{0}^{2 d}\right|^{+} \geq g$. If $g \geq 1$, its general member $D$ is smooth on $X-\{0\}$ and has an ordinary singularity of multiplicity $2 d$ at 0 . Thus $g_{C}=g$ by equation (5). If $g=0$ there exists an even divisor $D \in|L|$ with $2 \mu_{0}=$ mult $_{0} D \geq 2 d$. If $D$ admits at most ordinary singularities, Proposition 3.5 (respectively equation (5) ) gives

$$
\begin{aligned}
0 & \leq g_{C}=d^{2}-\sum_{x \in X_{2}^{-}(D)} \mu_{x}\left(\mu_{x}+1\right)-\sum_{x \in X_{2}^{+}(D)} \mu_{x}^{2}-\frac{1}{4} \sum_{j} r_{j}\left(r_{j}-1\right) \\
& \leq-\sum_{x \in X_{2}^{-}(D)} \mu_{x}\left(\mu_{x}+1\right)-\sum_{x \in X_{2}^{+}(D)-\{0\}} \mu_{x}^{2}-\frac{1}{4} \sum_{j} r_{j}\left(r_{j}-1\right) \leq 0 .
\end{aligned}
$$

Hence we must have $g_{C}=0$. In particular, $\mu_{x}=0$ for all $x \in X_{2}-\{0\}, \mu_{0}=d$, and $r_{j}=0$ or 1 . This implies that $D-\{0\}$ is smooth, and mult $_{0}(D)=2 d$. This proves assertion a) since, if $D$ would admit non-ordinary singularities, then the geometric genera of $D$ and $C$ would be even smaller, a contradiction.

The proof of $b$ ) is completely analogous.

\section{Singular Relations and Humbert Surfaces}

Let $(X, H)$ be a principally polarized abelian surface. Suppose that $(X, H)=$ $\left(X_{Z}, H_{0}\right)$ for some $Z \in \mathfrak{H}_{2}$. Then the rational representation $\rho_{r, Z}: \operatorname{End}(X) \longrightarrow$ $M_{4}(\mathbb{Z})$ and the analytic representation $\rho_{a, Z}: \operatorname{End}(X) \longrightarrow M_{2}(\mathbb{C})$ are related by

$$
\rho_{a, Z}(f)\left(Z, \mathbf{1}_{2}\right)=\left(Z, \mathbf{1}_{2}\right) \rho_{r, Z}(f)
$$


for all $f \in \operatorname{End}(X)$. Note that here both rational and analytic representation depend on the choice of $Z$. If $Z^{\prime}=M(Z)$, with $M \in \mathrm{Sp}_{4}(\mathbb{Z})$, is another element of $\mathfrak{H}_{2}$ representing $(X, H)$, then the corresponding rational representations are related by

$$
\rho_{r, M(Z)}={ }^{t} M^{-1} \rho_{r, Z}{ }^{t} M
$$

(see [CAV] Section 8.1). The principal polarization defines an isomorphism $\phi_{L_{0}}$ : $X \longrightarrow \operatorname{Pic}^{0}(X)=\hat{X}, \quad \phi_{H_{0}}(x):=t_{x}^{*} L_{0} \otimes L_{0}^{-1}$. The Rosati involution is the antiinvolution ' $: \operatorname{End}(X) \longrightarrow \operatorname{End}(X), \quad f^{\prime}:=\phi_{H_{0}}^{-1} \hat{f} \phi_{H_{0}}$. An element $f$ of $\operatorname{End}_{\mathbb{Q}}(X)$ is called symmetric, if $f^{\prime}=f$. Denote by $\operatorname{End}^{s}(X)$ the subgroup of symmetric endomorphisms.

Lemma 4.1. $f \in \operatorname{End}(X)$ is symmetric if and only if its rational representation is of the form $\rho_{r, Z}(f)=\left(\begin{array}{cc}A & B \\ C^{t} A\end{array}\right)$ with integral matrices $A=\left(\begin{array}{cc}a_{1} & a_{2} \\ a_{3} & a_{4}\end{array}\right), B=\left(\begin{array}{cc}0 & b \\ -b & 0\end{array}\right), C=$ $\left(\begin{array}{cc}0 & c \\ -c & 0\end{array}\right)$ whose coefficients satisfy

$$
a_{2} z_{1}+\left(a_{4}-a_{1}\right) z_{2}-a_{3} z_{3}+b\left(z_{2}^{2}-z_{1} z_{3}\right)+c=0 .
$$

Proof. The Rosati involution is the adjoint operator with respect to the alternating form $E_{0}=\operatorname{Im} H_{0}$ (see loc. cit. Prop. 5.1.1). In terms of matrices this means ${ }^{t} \rho_{r, Z}(f)\left(\begin{array}{cc}0 & \mathbf{1}_{2} \\ -\mathbf{1}_{2} & 0\end{array}\right)=\left(\begin{array}{cc}0 & \mathbf{1}_{2} \\ -\mathbf{1}_{2} & 0\end{array}\right) \rho_{r, Z}\left(f^{\prime}\right)$ for all $f \in \operatorname{End}(X)$. Now using equation (6) this implies the assertion.

The Lemma shows that $X=X_{Z}$ admits nontrivial symmetric endomorphisms if and only if the entries $z_{1}, z_{2}$ and $z_{3}$ of $Z$ satisfy a certain quadratic equation with integral coefficients. Now suppose conversely that $Z=\left(\begin{array}{ll}z_{1} & z_{2} \\ z_{2} & z_{3}\end{array}\right)$ satisfies the equation

$$
a z_{1}+b z_{2}+c z_{3}+d\left(z_{2}^{2}-z_{1} z_{3}\right)+e=0
$$

with integers $a, b, c, d$ and $e$. Setting

$$
R_{0}:=\left(\begin{array}{cccc}
0 & a & 0 & d \\
-c & b & -d & 0 \\
0 & e & 0 & -c \\
-e & 0 & a & b
\end{array}\right)
$$

then by Lemma 4.1 the matrices $n \mathbf{1}_{4}+m R_{0}$ are rational representations of symmetric endomorphisms of $X_{Z}$ for all $n, m \in \mathbb{Z}$.

Corollary 4.2. $Z=\left(\begin{array}{cc}z_{1} & z_{2} \\ z_{2} & z_{3}\end{array}\right)$ satisfies equation (8) if and only if $\rho_{r, Z}\left(\operatorname{End}^{s}\left(X_{Z}\right)\right)$ contains $R_{0}$.

Suppose $Z$ satisfies equation (8). Then $\operatorname{End}^{s}\left(X_{Z}\right)$ admits a symmetric endomorphism $f_{0}$ with $\rho_{r, Z}\left(f_{0}\right)=R_{0}$. Denoting by $P_{f}^{a}(t)$ the analytic characteristic polynomial $\operatorname{det}\left(t \mathbf{1}_{2}-\rho_{a, Z}(f)\right)$ of an endomorphism $f$, we get for the symmetric endomorphism $n_{X}+m f_{0}$ :

Proposition 4.3. The trace, norm and discriminant of $P_{n_{X}+m f_{0}}^{a}$ are:

$$
\begin{aligned}
\operatorname{Tr}_{a}\left(n_{X}+m f_{0}\right) & =2 n+m b, \\
\mathrm{~N}_{a}\left(n_{X}+m f_{0}\right) & =n^{2}+n m b+m^{2}(a c+d e), \quad \text { and } \\
\operatorname{Disc}\left(n_{X}+m f_{0}\right) & =m^{2}\left(b^{2}-4 a c-4 d e\right) .
\end{aligned}
$$


Proof. For any endomorphism $f, \operatorname{Tr}_{a}(f)$ is the trace of $\rho_{a, Z}(f), \mathrm{N}_{a}(f)=\operatorname{det} \rho_{a, Z}(f)$, and $\operatorname{Disc}(f)=\operatorname{Tr}_{a}(f)^{2}-4 \mathrm{~N}_{a}(f)$. Moreover, by equation (6), the analytic representation of $f_{0}$ is

$$
A_{Z}=\rho_{a, Z}\left(f_{0}\right)=\left(\begin{array}{cc}
-d z_{2} & d z_{1}-c \\
-d z_{3}+a & d z_{2}+b
\end{array}\right) .
$$

Using this and equation (8) implies the assertion.

Corollary 4.4. The subset $\left\{n_{X}+m f_{0} \mid m, n \in \mathbb{Z}\right\}$ of $\operatorname{End}^{s}\left(X_{Z}\right)$ is a ring isomorphic to $\mathbb{Z}[t] /\left(t^{2}-b t+a c+d e\right)$.

Proof. By Proposition 4.3 we have $P_{f_{0}}^{a}(t)=t^{2}-b t+a c+d e$. In particular, $f_{0}^{2}=$ $b f_{0}-a c-d e$. So $\left\{n_{X}+m f_{0} \mid m, n \in \mathbb{Z}\right\}$ is a ring and the map $\mathbb{Z}[t] \longrightarrow \operatorname{End}^{s}\left(X_{Z}\right)$, $t \mapsto f_{0}$ induces the above isomorphism.

Equation (8) is an equation on the Siegel upper half-space $\mathfrak{H}_{2}$. Pulling this equation back via the action of $\mathrm{Sp}_{4}(\mathbb{Z})$ gives a family of such equations. The following proposition shows that among these equations there is a uniquely determined normalized one.

Proposition 4.5. Suppose $Z=\left(\begin{array}{lll}z_{1} & z_{2} \\ z_{2} & z_{3}\end{array}\right)$ satisfies equation (8) and $\Delta:=b^{2}-4 a c-$ 4de. There is an $M \in \mathrm{Sp}_{4}(\mathbb{Z})$ such that $M(Z)=\left(\begin{array}{cc}z_{1}^{\prime} & z_{2}^{\prime} \\ z_{2}^{\prime} & z_{3}^{\prime}\end{array}\right)$ satisfies

$$
\begin{aligned}
-\frac{1}{4} \Delta z_{1}^{\prime}+z_{3}^{\prime}=0 \quad \text { if } \quad \Delta \equiv 0 \bmod 4 \\
\frac{1}{4}(1-\Delta) z_{1}^{\prime}+z_{2}^{\prime}+z_{3}^{\prime}=0 \quad \text { if } \quad \Delta \equiv 1 \bmod 4 .
\end{aligned}
$$

Proof. As in (91) let $R_{0}$ be the rational representation of the symmetric endomorphism defined by the singular relation. We only present a proof in the most general case, that is, if the integers $g_{0}, \ldots, g_{3}$ occurring in the subsequent steps are nonzero. According to equation (7) it remains to show that there is an $M \in \operatorname{Sp}_{4}(\mathbb{Z})$ and an integer $k$ such that ${ }^{t} M^{-1} R_{0}{ }^{t} M-k \mathbf{1}_{4}=\left(\begin{array}{cc}A & 0 \\ 0 & { }_{A}\end{array}\right)$ with $A=\left(\begin{array}{cc}0 & a^{\prime} \\ -1 & b^{\prime}\end{array}\right)$ with $b^{\prime}=0$ or 1. Then $\Delta=b^{\prime 2}+4 a^{\prime} \equiv b^{\prime} \bmod 4$ as required. The construction of $M$ proceeds in several steps:

Step I: Choose integers $\alpha$ and $\beta$ such that $\alpha e-\beta c=\operatorname{gcd}(e, c)=: g_{0}$. Then

$$
M_{0}:=\left(\begin{array}{cccc}
1 & 0 & 1 & 0 \\
0 & \alpha & 0 & \beta \\
-1 & 0 & 0 & 0 \\
0 & c / g_{0} & 0 & e / g_{0}
\end{array}\right) \in \operatorname{Sp}_{4}(\mathbb{Z})
$$

and $R_{1}:={ }^{t} M_{0}^{-1} R_{0}{ }^{t} M_{0}$ is of the form $\left(\begin{array}{cc}A_{1} & 0 \\ C_{1} & { }^{t} A_{1}\end{array}\right)$ with $A_{1}=\left(\begin{array}{cc}0 & a_{1} \\ -c_{1} & b_{1}\end{array}\right)$ and $C_{1}=$ $\left(\begin{array}{cc}0 & e_{1} \\ -e_{1} & 0\end{array}\right)$.

Step II: Since $\operatorname{gcd}\left(a_{1}, c_{1}, e_{1}\right) \mid g_{1}:=\operatorname{gcd}\left(a_{1}, e_{1}\right)$, Dirichlet's prime Theorem states that there is an integer $n$ such that $p:=\frac{e_{1}}{g_{1}}+n \frac{a_{1}}{g_{1}}$ is prime with $\left|c_{1}\right|<p$. Now

$$
M_{1}:=\left(\begin{array}{cc}
\mathbf{1}_{2} & \left(\begin{array}{cc}
-n & 0 \\
0 & 0 \\
0 & \mathbf{1}_{2}
\end{array}\right)
\end{array}\right)
$$

$\in \mathrm{Sp}_{4}(\mathbb{Z})$ and $R_{2}:={ }^{t} M_{1}^{-1} R_{0}{ }^{t} M_{1}=\left(\begin{array}{cc}A_{1} & 0 \\ C_{2}{ }^{t} A_{1}\end{array}\right)$, where $C_{2}:=\left(\begin{array}{cc}0 & e_{2} \\ -e_{2} & 0\end{array}\right)$ with $e_{2}=e_{1}+a_{1} n=g_{1} p$. In particular, $\operatorname{gcd}\left(c_{1}, e_{2}\right) \mid g_{1}$ (since $\left|c_{1}\right|<g_{1}$ ), and thus $\operatorname{gcd}\left(c_{1}, e_{2}\right) \mid a_{1}$. 
Step III: Choose integers $\gamma$ and $\delta$ such that $\gamma e_{2}-\delta c_{1}=\operatorname{gcd}\left(e_{2}, c_{1}\right):=g_{2}$. Then

$$
M_{2}:=\left(\begin{array}{cccc}
\gamma a_{1} / g_{2} & 0 & 1 & 0 \\
0 & \gamma & 0 & \delta \\
-1 & 0 & 0 & 0 \\
0 & c_{1} / g_{2} & 0 & e_{2} / g_{2}
\end{array}\right)
$$

$\in \mathrm{Sp}_{4}(\mathbb{Z})$ and $R_{3}:={ }^{t} M_{2}^{-1} R_{2}{ }^{t} M_{2}$ is of the form $\left(\begin{array}{cc}A_{3} & 0 \\ 0 & { }^{t} A_{3}\end{array}\right)$ with $A_{3}=\left(\begin{array}{cc}0 & a_{3} \\ -c_{3} & b_{3}\end{array}\right)$.

Step IV: Choose integers $\epsilon$ and $\eta$ such that $\epsilon a_{3}+\eta c_{3}=\operatorname{gcd}\left(a_{3}, c_{3}\right)=: g_{3}$. Then

$$
M_{3}:=\left(\begin{array}{cccc}
1 & 0 & 1 & 0 \\
0 & c_{3} / g_{3} & 0 & -a_{3} / g_{3} \\
-\epsilon a_{3} / g_{3} & 0 & \eta c_{3} / g_{3} & 0 \\
0 & \epsilon & 0 & \eta
\end{array}\right)
$$

$\in \mathrm{Sp}_{4}(\mathbb{Z})$ and $R_{4}:={ }^{t} M_{3}^{-1} R_{3}{ }^{t} M_{3}$ is of the form $\left(\begin{array}{cc}A_{4} & 0 \\ 0 & { }^{t} A_{4}\end{array}\right)$ with $A_{4}=\left(\begin{array}{cc}0 & a_{4} c_{4} \\ -c_{4} & b_{4}\end{array}\right)$ with $a_{4}, b_{4}, c_{4} \in \mathbb{Z}$.

Step $V$ : Since by assumption $\Delta$ is square-free the matrix $R_{4}$ is primitive and hence $\operatorname{gcd}\left(b_{4}, c_{4}\right)=1$. Choose integers $\mu$ and $\nu$ such that $\mu\left(\left(a_{4}+1\right) c_{4}+b_{4}\right)+\nu c_{4}=$ $\operatorname{gcd}\left(\left(a_{4}+1\right) c_{4}+b_{4}, c_{4}\right)=1$. Then

$$
M_{4}:=\left(\begin{array}{cccc}
1 & 0 & 1 & 0 \\
0 & c_{4} & 0 & -\left(a_{4}+1\right) c_{4}-b_{4} \\
-\mu\left(\left(a_{4}+1\right) c_{4}+b_{4}\right) & 0 & \nu c_{4} & 0 \\
0 & \mu & 0 & \nu
\end{array}\right)
$$

and

$$
M_{4}^{\prime}:=\left(\begin{array}{cccc}
1 & 0 & 0 & 0 \\
-1 & 1 & 0 & 0 \\
0 & 0 & 1 & 1 \\
0 & 0 & 0 & 1
\end{array}\right)
$$

are in $\operatorname{Sp}_{4}(\mathbb{Z})$ and $R_{5}:={ }^{t}\left(M_{4} M_{4}^{\prime}\right)^{-1} R_{4}{ }^{t}\left(M_{4} M_{4}^{\prime}\right)+c_{4} \mathbf{1}_{4}$ is of the form $\left(\begin{array}{cc}A_{5} & 0 \\ 0 & { }^{t} A_{5}\end{array}\right)$ with $A_{5}=\left(\begin{array}{cc}0 & a_{5} \\ -1 & b_{5}\end{array}\right)$.

Step VI: Let

$$
\tau:= \begin{cases}b_{5} / 2 & \text { if } b_{5} \text { is even } \\ \left(b_{5}-1\right) / 2 & \text { if } b_{5} \text { is odd }\end{cases}
$$

Then

$$
M_{5}:=\left(\begin{array}{cccc}
1 & 0 & 0 & 0 \\
\tau & 1 & 0 & 0 \\
0 & 0 & 1 & -\tau \\
0 & 0 & 0 & 1
\end{array}\right)
$$

$\in \mathrm{Sp}_{4}(\mathbb{Z})$ and $R_{6}:=^{t}\left(M_{5}\right)^{-1} R_{4}\left(M_{5}\right)-\tau \mathbf{1}_{4}$ is of the form $\left(\begin{array}{cc}A_{6} & 0 \\ 0 & { }^{t} A_{6}\end{array}\right)$ with $A_{6}=$ $\left(\begin{array}{cc}0 & a^{\prime} \\ -1 & b^{\prime}\end{array}\right)$ with $b^{\prime}=0$ if $b_{5}$ is even and $b^{\prime}=1$ if $b_{5}$ is odd.

This shows, in particular, that equation (8) induces an equation on the moduli space $\mathcal{A}_{2}$ which is uniquely determined by the discriminant $\Delta$. As a consequence we get (see also vdG Chapter IX Prop 2.3):

Corollary 4.6. For a principally polarized abelian surface $(X, H) \in \mathcal{A}_{2}$, the following statements are equivalent: 
i) $(X, H)=\left(X_{Z}, H_{0}\right)$ for some $Z=\left(\begin{array}{cc}z_{1} & z_{2} \\ z_{2} & z_{3}\end{array}\right)$ satisfying equation (8),

ii) $\operatorname{End}^{s}(X)$ contains a ring isomorphic to $\mathbb{Z}[t] /(p(t))$ where $p$ is a quadratic polynomial with discriminant $\Delta:=b^{2}-4 a c-4 d e$,

iii) $\operatorname{End}(X)$ contains a symmetric endomorphism $f_{\Delta}$ with discriminant $\Delta=$ $b^{2}-4 a c-4 d e$.

Note that in ii) we may assume equation (88) is normalized as in Proposition 4.5 and in iii) we may always choose $f_{\Delta}$ to be the endomorphism $f_{0}$ as defined above.

Following Humbert (see $[\mathrm{H}]$ ) we call an equation of the form

$$
a z_{1}+b z_{2}+c z_{3}+d\left(z_{2}^{2}-z_{1} z_{3}\right)+e=0
$$

with $a, b, c, d, e \in \mathbb{Z}$, a singular relation with invariant $\Delta=b^{2}-4 a c-4 d e$. According to Corollary 4.6 the singular relation (11) defines a subset of the moduli space of principally polarized abelian surfaces $\mathcal{A}_{2}=\mathfrak{H}_{2} / \mathrm{Sp}_{4}(\mathbb{Z})$ :

$\mathcal{H}_{\Delta}=\left\{(X, H) \in \mathcal{A}_{2} \mid(X, H)=\left(X_{Z}, H_{0}\right)\right.$ for some $Z \in \mathfrak{H}_{2}$ satisfying (111) $\}$.

Note that by Proposition 4.3 the invariant $\Delta$ is always $\equiv 0$ or $1 \bmod 4$.

Proposition 4.7. For any integer $\Delta \equiv 0$ or $1 \bmod 4$,

$$
\mathcal{H}_{\Delta}=\left\{(X, H) \in \mathcal{A}_{2} \mid \begin{array}{c}
\operatorname{End}(X) \text { contains a symmetric } \\
\text { endomorphism with discriminant } \Delta
\end{array}\right\} .
$$

Moreover, $\mathcal{H}_{\Delta}$ is a surface, for $\Delta>0, \mathcal{H}_{0}=\mathcal{A}_{2}$, and $\mathcal{H}_{\Delta}=\emptyset$, if $\Delta<0$.

In particular, $\mathcal{H}_{\Delta}$ is uniquely determined by $\Delta$. For $\Delta>0, \mathcal{H}_{\Delta}$ is called a Humbert surface (with invariant $\Delta$ ).

Proof. The first characterization of $\mathcal{H}_{\Delta}$ follows from Corollary 4.6 iii). Suppose $(X, H) \in \mathcal{H}_{\Delta}$. Let $f$ be a symmetric endomorphism of $X$ with discriminant $\Delta$ and $A$ its analytic representation. The Rosati involution is the adjoint operator for the Hermitian form $H$ (see loc. cit. Prop. 5.1.1) implying that $H:=H_{0}(A \cdot, \cdot)$ is Hermitian. In particular, all eigenvalues of $A$ are real; namely, if $\lambda$ is an eigenvalue with eigenvector $v$, then $H(v, v)=\lambda H_{0}(v, v)$. This implies that $\mathcal{H}_{\Delta}$ is nonempty if and only if $\Delta=\Delta(f) \geq 0$. If $\Delta=0$, then $f$ is a multiple of the identity on $X$ and hence $\mathcal{H}_{\Delta}=\mathcal{A}_{2}$.

Obviously we have $\mathcal{H}_{\Delta} \subseteq \mathcal{H}_{m^{2} \Delta}$ for any positive integer $m$. If $\Delta$ is a square we moreover have

Proposition 4.8. Let $\delta$ be a positive integer. The Humbert surface $\mathcal{H}_{\delta^{2}}$ is the locus of principally polarized abelian surfaces $\left(X, L_{0}\right) \in \mathcal{A}_{2}$ admitting an isogeny of degree $\delta^{2}$,

$$
\left(E_{1} \times E_{2}, p_{1}^{*} \mathcal{O}_{E_{1}}(\delta) \otimes p_{2}^{*} \mathcal{O}_{E_{2}}(\delta)\right) \longrightarrow\left(X, L_{0}\right) .
$$

Here $\mathcal{O}_{E_{i}}(\delta)$ indicates a line bundle of degree $\delta$ on the elliptic curve $E_{i}$ and $p_{i}: E_{1} \times E_{2} \longrightarrow E_{i}$ is the natural projection.

Proof. Suppose $\left(X, L_{0}\right) \in \mathcal{H}_{\delta^{2}}$ for some $\delta>0$. Then $\operatorname{End}(X)$ contains a symmetric endomorphism $f_{\delta^{2}}$ with discriminant $\delta^{2}$. In particular, the eigenvalues of its analytic representation are integers, say $\lambda$ and $\mu$. Since $0<\delta^{2}=(\lambda-\mu)^{2}$ we have $\lambda \neq \mu$ and $f_{\delta^{2}}-\mu_{X}$ is an endomorphism with eigenvalues 0 and $\delta=\lambda-\mu$. Hence its image $E_{1}:=\operatorname{im}\left(f_{\delta^{2}}-\mu_{X}\right)$ is an elliptic curve in $X$. Since $P_{f_{\delta^{2}}-\mu_{X}}^{a}(t)=t^{2}-\delta t$, the endomorphism $f_{\delta^{2}}-\mu_{X}$ is the norm-endomorphism of $E_{1}$ in $X$ and $\operatorname{deg} L_{0} \mid E_{1}=\delta$. 
Moreover, $X$ admits a second elliptic curve $E_{2}$ with $\operatorname{deg} L_{0} \mid E_{2}=\delta$ such that the addition induces an isogeny $\left(E_{1} \times E_{2}, p_{1}^{*} L_{0}\left|E_{1} \otimes p_{2}^{*} L_{0}\right| E_{2}\right) \longrightarrow\left(X, L_{0}\right)$ of degree $\delta^{2}$ (see [CAV] Sections 5.3 and 12.1).

Conversely suppose there is an isogeny as in (12). Then by loc. cit., Lemma 5.3.1 the norm-endomorphism $f=N_{E_{1}}$ of $\operatorname{im}\left(E_{1}\right)$ in $X$ is a symmetric endomorphism of $X$ satisfying $f^{2}-\delta^{\prime} f=0$ with some divisor $\delta^{\prime}$ of $\delta$. So $\operatorname{Disc}(f)=\operatorname{Disc}\left(t^{2}-\delta^{\prime} t\right)=$ $\left(\delta^{\prime}\right)^{2}$ and $\left(X, L_{0}\right) \in \mathcal{H}_{\left(\delta^{\prime}\right)^{2}} \subseteq \mathcal{H}_{\delta^{2}}$.

Given a principally polarized abelian surface $\left(X, L_{0}\right)$, there is an isomorphism

$$
\Phi: \mathrm{NS}(X) \longrightarrow \operatorname{End}^{s}(X), L \mapsto \phi_{L_{0}}^{-1} \phi_{L}
$$

(see [CAV] Proposition 5.2.1). In particular, $\mathrm{NS}(X)$ and $\operatorname{End}^{s}(X)$ are of the same rank $\rho(X)$.

Proposition 4.9. Suppose $\Delta$ and $\Delta^{\prime}$ are nonsquare, positive integers $\equiv 0$ or $1 \mathrm{mod}$ 4 and $\Delta$ is not a square.

(1) $\left(X, L_{0}\right) \in \mathcal{A}_{2}-\bigcup_{\delta>0} \mathcal{H}_{\delta^{2}}$ if and only if $X$ is simple.

(2) If $\left(X, L_{0}\right) \in \mathcal{H}_{\Delta}$, then $\operatorname{End}^{s}(X) \otimes_{\mathbb{Z}} \mathbb{Q}$ contains the real quadratic number field $\mathbb{Q}(\sqrt{\Delta})$ and $\rho(X) \geq 2$. For the general member of $\mathcal{H}_{\Delta}$, equality holds in both statements.

(3) If $\left(X, L_{0}\right) \in \mathcal{H}_{\Delta} \cap \mathcal{H}_{\Delta^{\prime}}$, then $X$ is either simple, $\operatorname{End}_{\mathbb{Q}}(X)$ is a totally indefinite quaternion algebra, and $\rho(X)=3$, or $X$ is isomorphic to a product $E \times E$ with an elliptic curve $E$, and $\rho(X) \geq 3$.

Proof. (1) is a consequence of Proposition 4.8,

As for (2): By what we have said above we necessarily have $\rho(X) \geq 2$. By Corollary [4.6] ii), $\operatorname{End}^{s}(X) \otimes_{\mathbb{Z}} \mathbb{Q}$ contains $(\mathbb{Z}[t] / p(t)) \otimes_{\mathbb{Z}} \mathbb{Q}$ with a quadratic polynomial $p \in \mathbb{Z}[t]$ such that $\operatorname{Disc}(p)=\Delta$. But this equals the real quadratic number field $\mathbb{Q}(\sqrt{\Delta})$, the invariant $\Delta$ being nonsquare. The last assertion is obvious.

As for (3): By $(2) \operatorname{End}^{s}(X) \otimes_{\mathbb{Z}} \mathbb{Q}$ contains both number fields $\mathbb{Q}(\sqrt{\Delta})$ and $\mathbb{Q}\left(\sqrt{\Delta^{\prime}}\right)$. So $\rho(X) \geq 3$ and thus $X$ is either simple and $\operatorname{End}^{s}(X) \otimes_{\mathbb{Z}} \mathbb{Q}$ is an indefinite quaternion algebra over $\mathbb{Q}$, or $X$ is isomorphic to a product $E \times E$ with an elliptic curve $E$ (see for example [CT] Proposition 2.7.1).

\section{Line Bundles Associated with Symmetric Endomorphisms}

In this section we translate properties of symmetric endomorphisms associated with singular relations into terms of line bundles.

Suppose $\Delta$ is a positive integer and $(X, H)=\left(X_{Z}, H_{0}\right) \in \mathcal{H}_{\Delta}$. According to Proposition 4.5 we may assume that $Z$ satisfies the singular relation $a z_{1}+b z_{2}+$ $z_{3}=0$ with $\Delta=b^{2}-4 a$ and $b=0$ or 1 . Then $\operatorname{End}^{s}(X)$ contains a symmetric endomorphism $f_{\Delta}$ with rational representation $\rho_{r, Z}\left(f_{\Delta}\right)=\left(\begin{array}{cc}A & 0 \\ 0 & t_{A}\end{array}\right), A=\left(\begin{array}{cc}0 & a \\ -1 & b\end{array}\right)$. Note that by (10) the analytic representation is $\rho_{a, Z}\left(f_{\Delta}\right)={ }^{t} A$.

Via the isomorphism $\Phi: \mathrm{NS}(X) \longrightarrow \operatorname{End}^{s}(X)$, see (13), the endomorphism $f_{\Delta}$ defines a Hermitian form $H_{\Delta} \in \mathrm{NS}(X)$. Let $L_{0}$ and $L_{\Delta}$ be symmetric line bundles on $X$ with $c_{1}\left(L_{0}\right)=H_{0}$ and $c_{1}\left(L_{\Delta}\right)=H_{\Delta}$. In these terms the line bundle $L_{0}^{n} \otimes L_{\Delta}^{m}$, $n, m \in \mathbb{Z}$, corresponds to the symmetric endomorphism $n_{X}+m f_{\Delta}$. 
Lemma 5.1. Let $L$ be a line bundle algebraically equivalent to $L_{0}^{n} \otimes L_{\Delta}^{m}$, with $n, m \in \mathbb{Z}$. Then

$$
\begin{aligned}
\left(L_{0} \cdot L\right) & =\operatorname{Tr}_{a}\left(f_{L}\right)=2 n+m b, \\
\frac{1}{2}\left(L^{2}\right) & =\mathrm{N}_{a}\left(f_{L}\right)=n^{2}+n m b+m^{2} a .
\end{aligned}
$$

Proof. This is a consequence of Proposition 4.3 and the fact that the coefficients of the analytic characteristic polynomial of a symmetric endomorphism are given by the intersection numbers of its associated line bundle (see [CAV] Proposition $5.2 .3)$.

This gives a new characterization of the Humbert surface $\mathcal{H}_{\Delta}$ :

Corollary 5.2. A principally polarized abelian surface $\left(X, L_{0}\right)$ belongs to the Humbert surface $\mathcal{H}_{\Delta}$ if and only if $X$ admits a line bundle $L_{\Delta}$ satisfying $\left(L_{0} \cdot L_{\Delta}\right)^{2}-$ $2\left(L_{\Delta}^{2}\right)=\Delta$. Moreover, $L_{\Delta}$ can be chosen in such a way that $\left(L_{0} \cdot L_{\Delta}\right)=b$ and $\left(L_{\Delta}^{2}\right)=\frac{b-\Delta}{2}$ where $b \in\{0,1\}$ such that $b \equiv \Delta \bmod 4$.

Proof. The equivalence is a consequence of Proposition 4.7 and the previous Lemma. Now suppose $(X, H) \in \mathcal{H}_{\Delta}$. As outlined at the beginning of this section and in Lemma 5.1 there is a line bundle $L_{\Delta}$ with $\left(L_{0} \cdot L_{\Delta}\right)=b$ and $\left(L_{\Delta}^{2}\right)=2 a=\frac{b^{2}-\Delta}{2}=$ $\frac{b-\Delta}{2}$.

For the type of line bundles $L \equiv L_{0}^{n} \otimes L_{\Delta}^{m}$ we have

Lemma 5.3. $L$ is of type $\left(\operatorname{gcd}(n, m), \frac{n^{2}+n m b+m^{2} a}{\operatorname{gcd}(n, m)}\right)=\left(\operatorname{gcd}(n, m), \frac{\left(L^{2}\right)}{2 \operatorname{gcd}(n, m)}\right)$.

Proof. Denote by $f_{L}=n_{X}+m f_{\Delta}$ the endomorphism corresponding to $L$. By definition of $\Phi$ we have $H_{L}:=c_{1}(L)=H_{0}\left(\rho_{a, Z}\left(f_{L}\right) \cdot, \cdot\right)$. So the matrix of its imaginary part is $\left(\begin{array}{cc}{ }^{t} D & 0 \\ 0 & D\end{array}\right)\left(\begin{array}{cc}0 & \mathbf{1}_{2} \\ -\mathbf{1}_{2} & 0\end{array}\right)=\left(\begin{array}{cc}0 & { }^{t} D \\ -D & 0\end{array}\right)$ with $D={ }^{t} \rho_{a, Z}\left(f_{L}\right)=n \mathbf{1}_{2}+m A=$ $\left(\begin{array}{cc}n & m a \\ -m & n+m b\end{array}\right)$. If $\left(d_{1}, d_{2}\right)$ are its elementary divisors, then $d_{1}$ is the largest positive integer such that $\frac{1}{d_{1}} D$ is integral and $d_{2}=\frac{1}{d_{1}} \operatorname{det} D$. This implies the assertion.

\section{The Kummer Plane Associated with $\left(X, L_{0}\right)$}

Consider an irreducible principally polarized abelian surface $\left(X, L_{0}\right)$ and the Kummer map $\varphi: X \longrightarrow K_{X} \subset \mathbb{P}_{3}$. The $16_{6}$ configuration (see Proposition 2.2) states that there are 6 singular planes $P_{1}, \ldots, P_{6}$ containing the singular point $0=\varphi(0)$. Consider the linear projection

$$
\pi: \mathbb{P}_{3}-\{0\} \longrightarrow \mathbb{P}_{2}
$$

with center 0 . The singular planes $P_{i}$ map to lines $l_{i}, i=1, \ldots, 6$, called singular lines. Since by Proposition 2.3 any two singular planes have exactly two singular points in common, the intersection of two lines $l_{i} \cap l_{j}$ is the image of the singular point in $P_{i} \cap P_{j}$ different from 0 . So in $\mathbb{P}_{2}$ the 15 points $l_{i} \cap l_{j}, 1 \leq i<j \leq 6$ are in one-to-one correspondence to the 15 singular points of $K_{X}$ different from 0 . We call $\left(\mathbb{P}_{2}, l_{1}, \ldots, l_{6}\right)$ the Kummer plane associated to $\left(X, L_{0}\right)$.

The configuration of lines and points in $\mathbb{P}_{2}$ is indicated in the following picture. 


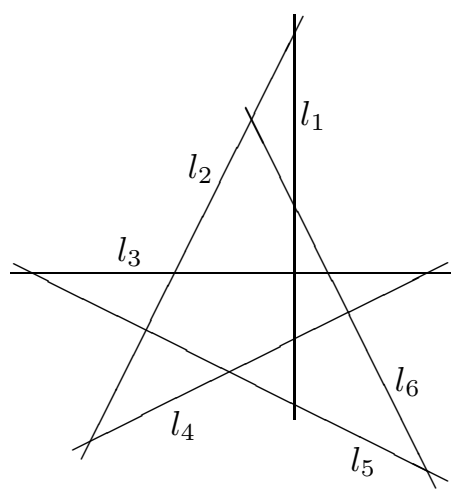

Next we study curves on the Kummer plane $\left(\mathbb{P}_{2}, l_{1}, \ldots, l_{6}\right)$ associated to the principally polarized abelian surface $\left(X, L_{0}\right)$. For a curve $C$ on $K_{X}$ denote by $C^{\prime}=\overline{\pi(C)-\{0\}}$ the closure of its image in $\mathbb{P}_{2}$. Then the projection (14) induces a natural map $\pi: C \longrightarrow C^{\prime}$, which by abuse of notation we denote by the same symbol.

Lemma 6.1. Suppose $C$ is a curve on $K_{X}$ and $x \in C^{\prime} \cap l_{i}$ for $i \in\{1, \ldots, 6\}$. Then either $x \in\left\{l_{i} \cap l_{j}\right\}$ or $\left(C^{\prime} \cdot l_{i}\right)_{x}$ is even.

Proof. This is a direct consequence of the fact that singular planes touch the Kummer surface along double conics.

Lemma 6.2. Suppose $C$ is a curve of degree $\delta$ on $K_{X}$ and $D=\varphi^{*} C$ on $X$. If $\delta=4 n$ and $D \in\left|L_{0}^{2 n}\right|$, then the natural map $\pi: C \longrightarrow C^{\prime}$ is of degree $\leq 2$. In any other case $\pi: C \longrightarrow C^{\prime}$ is birational and $\operatorname{deg} C^{\prime}=\left(D \cdot L_{0}\right)-\operatorname{mult}_{0}(D)$.

Proof. Since $\operatorname{deg} K_{X}=4$ and the center of projection is a double point of $K_{X}$, the map $\pi: C \longrightarrow C^{\prime}$ is either of degree 1 or 2 . Obviously it is of degree 2 only if $C$ is a complete intersection. By Proposition 2.1 this is the case if and only if $D \in\left|L_{0}^{2 n}\right|$ for some $n$. Note that the degree of a complete intersection is divisible by 4. This implies the first assertion. As for the second assertion note that mult $_{0}(C)=$ mult $_{0}(D)$ by Lemma 3.1 and $\operatorname{deg} C=\left(C \cdot \mathcal{O}_{\mathbb{P}_{3}}(1)\right)=\frac{1}{2}\left(D \cdot L_{0}^{2}\right)=\left(D \cdot L_{0}\right)$. Now the assertion follows from $\operatorname{deg} C^{\prime}=\operatorname{deg} C-\operatorname{mult}_{0}(C)$.

Proposition 6.3. Suppose $C^{\prime}$ is a curve of degree $\delta \geq 1$ on the Kummer plane $\left(\mathbb{P}_{2}, l_{1}, \ldots, l_{6}\right)$ of $\left(X, L_{0}\right)$. If $C^{\prime}$ intersects the lines $l_{i}$ properly such that every $x \in C^{\prime} \cap l_{i}, i=1, \ldots, 6$ satisfies either $x \in\left\{l_{i} \cap l_{j}\right\}$ or $\left(C^{\prime} \cdot l_{i}\right)_{x}$ is even, then

a) there is a curve $C$ on $K_{X}$ mapping birationally to $C^{\prime}$ via $\pi$;

b) $\delta \leq \operatorname{deg} C \leq 2 \delta$;

c) mult $_{\pi(p)} C^{\prime}=$ mult $_{p} C$ for all $p \in C-\{0\}$.

Proof. According to $\mathrm{CAV}$ Section 10.3 we may choose homogenous coordinates $z_{0}, \ldots, z_{3}$ in such a way that 1$)$ the coordinate points $(1: 0: 0: 0),(0: 1: 0:$ $0),(0: 0: 1: 0)$ and $(0: 0: 0: 1)$ are among the singular points of $K_{X}$ and $2)$ the coordinate planes $\left\{z_{i}=0\right\}$ are among the singular planes. Moreover, we may assume that the origin 0 maps to the coordinate point $(0: 0: 0: 1)$. In particular, $\varphi(0)$ is not contained in $\left\{z_{3}=0\right\}$ and thus we may identify the Kummer plane with $\mathbb{P}_{2}=\mathbb{P}\left(z_{0}: z_{1}: z_{2}\right)=\left\{z_{3}=0\right\}$. The equation of $K_{X}$ is of the form $f_{2} z_{3}^{2}+2 f_{3} z_{3}+f_{4}=0$ with homogenous polynomials $f_{i}$ in $z_{0}, z_{1}, z_{2}$ of degree 
$\operatorname{deg} f_{i}=i$. By [GD] Theorem 2.6, $\left\{f_{3}^{2}-f_{2} f_{4}=0\right\} \subset \mathbb{P}_{3}=\mathbb{P}\left(z_{0}: z_{1}: z_{2}: z_{3}\right)$ is the union of the 6 singular planes $P_{1}, \ldots, P_{6}$ or equivalently $\left\{f_{3}^{2}-f_{2} f_{4}=0\right\}=\bigcup_{i-1}^{6} l_{i}$ in $\mathbb{P}_{2}=\mathbb{P}\left(z_{0}: z_{1}: z_{2}\right)$. By assumption $x \in C^{\prime} \cap l_{i}$ is either a double point of $\bigcup_{i-1}^{6} l_{i}$ or $\left(C^{\prime} \cdot l_{i}\right)_{x}$ is even. Hence $\bigcup_{i-1}^{6} l_{i} \mid C^{\prime}=2 D^{\prime}$ with a divisor $D^{\prime}$ of degree $3 \delta$ on $C^{\prime}$.

If $\delta \geq 3$ choose an $\alpha \in H^{0}\left(\mathcal{O}_{\mathbb{P}_{2}}(2(\delta-3))\right)$ and consider the divisor $A:=\{\alpha=$ $0\} \mid C^{\prime}$ on $C^{\prime}$. Since $h^{0}\left(\mathcal{O}_{\mathbb{P}_{2}}(2 \delta-3)\right)-\operatorname{deg} A-\operatorname{deg} D^{\prime}=\left(2 \delta^{2}-3 \delta+1\right)-2(\delta-3) \delta-3 \delta=$ 1 , there is a $\beta^{\prime} \in H^{0}\left(\mathcal{O}_{\mathbb{P}_{2}}(2 \delta-3)\right)$ such that $\left\{\beta^{\prime}=0\right\} \mid C^{\prime}$ contains the divisor $A+D^{\prime}$. If $\delta=1$ or 2 take $\alpha=1$ and $\beta^{\prime} \in H^{0}\left(\mathcal{O}_{\mathbb{P}_{2}}(3)\right)$ such that $D^{\prime} \subset\left\{\beta^{\prime}=0\right\} \mid C^{\prime}$. This setting defines a pencil of curves of degree $2(2 \delta-3)$ in $\mathbb{P}_{2}$ :

$$
F_{t}=\left\{\alpha^{2}\left(f_{3}^{2}-f_{2} f_{4}\right)-t \beta^{2}=0\right\}, \quad t \in \mathbb{C} .
$$

It is easy to see using $\left(F_{t} \cdot C^{\prime}\right)=2(2 \delta-3) \delta=2 \operatorname{deg}\left(A+D^{\prime}\right)$ that

$$
F_{t} \mid C^{\prime}=2 A+2 D^{\prime}
$$

for all $t \in \mathbb{C}$. Choosing $t_{0} \in \mathbb{C}$ such that the curve $F_{t_{0}}$ contains a further point of $C^{\prime}$ we conclude that $C^{\prime} \subset F_{t_{0}}$. In terms of equations this means

$$
g \mid\left(\alpha^{2}\left(f_{3}^{2}-f_{2} f_{4}\right)-\beta^{2}\right)
$$

where $C^{\prime}=\{g=0\}$ and $\beta=\sqrt{t_{0}} \beta^{\prime}$.

Consider the curves $C_{ \pm}:=\left\{\alpha\left(f_{2} z_{3}+f_{3}\right) \pm \beta=0\right\} \cap \pi^{-1}\left(C^{\prime}\right)$ in $\mathbb{P}_{3}$. An immediate computation shows that

$$
C_{+} \cup C_{-}=\left\{\left\{\alpha^{2} f_{2}=0\right\} \cup K_{X}\right\} \cap \pi^{-1}\left(C^{\prime}\right) .
$$

Note that $\left\{\alpha^{2} f_{2}=0\right\} \cap \pi^{-1}\left(C^{\prime}\right)=\pi^{-1}\left(\left\{\alpha^{2} f_{2}=0\right\} \cap C^{\prime}\right)$ is the union of $\operatorname{deg} \alpha^{2} f_{2}$. $\operatorname{deg} \pi^{-1}\left(C^{\prime}\right)=4 \delta(\delta-3)+2 \delta$ lines. These are exactly the lines connecting the points $\left\{\alpha^{2} f_{2}=0\right\} \cap C^{\prime} \subset \mathbb{P}_{2}=\left\{z_{3}=0\right\}$ with $(0: 0: 0: 1) \in \mathbb{P}_{3}$. Here the $2 \delta(\delta-3)$ lines $\pi^{-1}\left(\{\alpha=0\} \cap C^{\prime}\right)$ occur with multiplicity 2 and each curve $C_{+}$and $C_{-}$contains a copy of these lines. The remaining $2 \delta$ lines $\pi^{-1}\left(\left\{f_{2}=0\right\} \cap C^{\prime}\right)$ are distributed somehow between $C_{+}$and $C_{-}$. So $C_{+} \cup C_{-}-\pi^{-1}\left(\left\{\alpha^{2} f_{2}=0\right\} \cap C^{\prime}\right)=K_{X} \cap \pi^{-1}\left(C^{\prime}\right)$ is a union of two curves $C_{1} \cup C_{2}$ whose degrees satisfy

$$
\operatorname{deg} C_{1}+\operatorname{deg} C_{2}=\operatorname{deg}\left(K_{X} \cap \pi^{-1}\left(C^{\prime}\right)\right)=4 \delta
$$

and

$$
\begin{aligned}
\delta=\operatorname{deg}\left(C_{ \pm}-\pi^{-1}\left(\left\{\alpha f_{2}=0\right\} \cap C^{\prime}\right)\right) & \leq \operatorname{deg} C_{i} \\
& \leq \operatorname{deg}\left(C_{ \pm}-\pi^{-1}\left(\{\alpha=0\} \cap C^{\prime}\right)\right)=3 \delta .
\end{aligned}
$$

So either $C_{1}$ or $C_{2}$ satisfies assertion b). Take $C$ to be this curve. Since obviously $\pi$ projects both curves $C_{ \pm}$birationally to $C^{\prime}$, so the same holds for $C$.

As for c) note that if $C^{\prime}$ is smooth in $\pi(p)$, then $C$ is smooth in $p$, since the multiplicity at a point is by definition the degree of the tangent cone at this point and $\pi$ is a linear projection.

The following example illustrates Proposition 6.3 and its proof.

Example 1. Let the notation be as in the proof above. Consider the conic $C$ on $K_{X}$ defined by $2 C=K_{X} \cap\left\{z_{3}=0\right\}$ and its (birational!) image $C^{\prime}$ in $\mathbb{P}_{2}$. Note that the term $f_{4}$ in the equation of $K_{X}$ is a square, say $f_{4}=F_{2}^{2}$; so $C=\left\{z_{3}=F_{2}=0\right\}$ and $C^{\prime}=\left\{F_{2}=0\right\} \subset \mathbb{P}_{2}$. Then we get with $\alpha=1$ and $\beta=f_{3}$,

$$
C_{+}=\left\{f_{2} z_{3}+2 f_{3}=F_{2}=0\right\}
$$


and

$$
\begin{aligned}
C_{-} & =\left\{f_{2} z_{3}=F_{2}=0\right\}=\left\{f_{2}=F_{2}=0\right\} \cup\left\{z_{3}=F_{2}=0\right\} \\
& =L_{1} \cup \cdots L_{4} \cup C .
\end{aligned}
$$

In particular, all 4 lines $L_{1}, \ldots, L_{4}$ are contained in the curve $C_{-}$. So $C_{1}=C_{-}-$ $L_{1}-\cdots-L_{4}=C$, which is of degree 2 and $C_{2}=C_{+}$is of degree 6 . Moreover, we have

$$
\begin{aligned}
K_{X} \cap \pi^{-1}\left(C^{\prime}\right) & =\left\{f_{2} z_{3}^{2}+2 f_{3} z_{3}+F_{2}^{2}=F_{2}=0\right\} \\
& =\left\{z_{3} \cdot\left(f_{2} z_{3}+2 f_{3}\right)=F_{2}=0\right\} \\
& =\left\{z_{3}=F_{2}=0\right\} \cup\left\{f_{2} z_{3}+2 f_{3}=F_{2}=0\right\} \\
& =C \cup C_{+} .
\end{aligned}
$$

Note that there are two complete intersections containing the conic $C$ :

$$
K_{X} \cap\left\{z_{3}=0\right\}=2 C \text { and } K_{X} \cap\left\{F_{2}=0\right\}=C \cup C_{+} .
$$

Proposition 6.4. Suppose the Kummer plane $\left(\mathbb{P}_{2}, l_{1}, \ldots, l_{6}\right)$ of $\left(X, L_{0}\right)$ admits a curve $C^{\prime}$ of (geometric) genus $g$ and degree $\delta$ satisfying a) $\# C^{\prime} \cap\left\{l_{i} \cap l_{j}\right\}=\kappa$ and these points are smooth points of $C^{\prime}$, and b) for any other point $x \in C^{\prime}$ the multiplicity $\left(C^{\prime} \cdot l_{i}\right)_{x}$ is even. Then $X$ admits a line bundle $L$ with

$$
\left(L \cdot L_{0}\right)^{2}-2\left(L^{2}\right) \leq \begin{cases}2 \delta^{2}-2 \kappa-8 g+8 & \text { if } \kappa \equiv \delta \bmod 2, \\ 2 \delta^{2}-2 \kappa-8 g+7 & \text { if } \kappa \neq \equiv \delta \bmod 2 .\end{cases}
$$

Notice that the upper bound is $\equiv 0 \bmod 4$ if $\kappa \equiv \delta \bmod 2$ and $\equiv 1 \bmod 4$ if $\kappa \not \equiv \delta \bmod 2$.

Proof. Let $C$ be the curve on $K_{X}$ projecting birationally to $C^{\prime}$ as in Lemma 6.3 and denote $D=\varphi^{*} C$ and $L=\mathcal{O}_{X}(D)$. Note that with $C^{\prime}$ also $C$ is of genus $g$. If $x_{1}, \ldots, x_{\kappa}$ denote the 2 -torsion points lying over the points $l_{i} \cap l_{j} \cap C^{\prime}$, then mult $_{x_{i}}(D)=1$ for $i=1, \ldots, \kappa$ by Proposition $6.3 \mathrm{c}$ ). In particular, $\left\{x_{1}, \ldots, x_{\kappa}\right\} \subset$ $X_{2}^{-}(D)$. Since the cardinality of $X_{2}^{ \pm}(D)$ is always even, we have

$$
X_{2}^{-}(D)= \begin{cases}\left\{x_{1}, \ldots, x_{\kappa}\right\} & \text { if } \kappa \text { is even, } \\ \left\{0, x_{1}, \ldots, x_{\kappa}\right\} & \text { if } \kappa \text { is odd. }\end{cases}
$$

In particular, $D$ is even (respectively odd) if $\kappa$ is even (respectively odd). Let $\operatorname{mult}_{0}(D)=2 \mu$ if $\kappa$ is even and $\operatorname{mult}_{0}(D)=2 \mu+1$ if $\kappa$ is odd for some $\mu \geq 0$. Note that $C$ and $D$ do not pass through the remaining 2-torsion points; so $\operatorname{mult}_{x}(D)=0$ for $x \in X_{2}^{+}(D)-\{0\}$. By Lemma 6.2 we have

$$
\left(L \cdot L_{0}\right)=\operatorname{deg} C=\operatorname{deg} C^{\prime}+\operatorname{mult}_{0}(D)= \begin{cases}\delta+2 \mu & \text { if } \kappa \text { is even, } \\ \delta+2 \mu+1 & \text { if } \kappa \text { is odd }\end{cases}
$$

Combining this with Proposition 6.3 b) we obtain

$$
2 \mu \leq \begin{cases}\delta & \text { if } \kappa \text { is even, } \\ \delta-1 & \text { if } \kappa \text { is odd }\end{cases}
$$


By Proposition 3.5 and Lemma 1.2

$$
g=g_{C}= \begin{cases}\frac{1}{4}\left(\left(L^{2}\right)-\kappa\right)+1-\mu^{2}-\nu & \text { if } \kappa \text { is even, } \\ \frac{1}{4}\left(\left(L^{2}\right)-\kappa-1\right)+1-\mu(\mu+1)-\nu & \text { if } \kappa \text { is odd }\end{cases}
$$

where $\nu=\frac{1}{4} \sum_{j} r_{j}\left(r_{j}-1\right)$, which is a positive integer. Hence

$$
\left(L^{2}\right)= \begin{cases}\kappa-4+4 \mu^{2}+8 g+4 \nu & \text { if } \kappa \text { is even, } \\ \kappa-3+4 \mu(\mu+1)+8 g+4 \nu & \text { if } \kappa \text { is odd. }\end{cases}
$$

Now an immediate computation gives

$$
\begin{aligned}
\left(L \cdot L_{0}\right)^{2}-2 & \left(L^{2}\right) \\
& = \begin{cases}\delta^{2}+4 \mu(\delta-\mu)-2 \kappa-8 g+8-8 \nu & \text { if } \kappa \text { is even, } \\
\delta^{2}+2 \delta+4 \mu(\delta-\mu-1)-2 \kappa-8 g+7-8 \nu & \text { if } \kappa \text { is odd. }\end{cases}
\end{aligned}
$$

Now an easy computation using $\nu \geq 0$ and the estimate of $\mu$, and distinguishing the cases where $d$ and $\mu$ are even or odd, implies the assertion.

\section{Characterization of Humbert Surfaces \\ in Terms of the Kummer Plane}

In this Section we present a geometric characterization of all Humbert surfaces in terms of curves on the associated Kummer planes. First we treat the case of rational curves $C^{\prime}$ in $\mathbb{P}_{2}$. One of the crucial tools for this is Proposition 6.4 Therefore we need to distingush the following cases:

$$
\begin{aligned}
\Delta & =\left\{\begin{array}{l}
8 d^{2}+9-2 k \\
8 d(d+1)+9-2 k
\end{array} \quad \text { (then } \Delta \equiv 1 \bmod 4\right), \\
\Delta & =\left\{\begin{array}{l}
8 d^{2}+8-2 k \\
8 d(d+1)+12-2 k
\end{array} \quad(\text { then } \Delta \equiv 0 \bmod 4)\right.
\end{aligned}
$$

with $d \geq 1$ and $k \in\{4,6,8,10,12\}$. Note that these are exactly the values occurring as upper bounds in Proposition 6.4 setting $\delta=2 d$ or $2 d+1$ and $\kappa=k$ or $k-1$. For sake of comprehensibility we treat these four cases separately. Note that these values do not cover all positive integers $\equiv 0$ or $1 \bmod 4$.

Theorem 7.1. Suppose $\Delta=8 d^{2}+9-2 k$ with $d \geq 1$ and $k \in\{4,6,8,10,12\}$. If $\left(X, L_{0}\right) \in \mathcal{H}_{\Delta}$ is an irreducible principally polarized abelian surface, then the associated Kummer plane $\left(\mathbb{P}_{2}, l_{1}, \ldots, l_{6}\right)$ admits a rational curve $C^{\prime}$ of degree $2 d$ passing smoothly through exactly $k-1$ points of $\left\{l_{i} \cap l_{j}\right\}$ and touching the singular lines $l_{i}$ in the remaining intersection points with even multiplicity. Conversely, if $\left(\mathbb{P}_{2}, l_{1}, \ldots, l_{6}\right)$ admits such a curve, then $\left(X, L_{0}\right) \in \mathcal{H}_{\Delta^{\prime}}$ with $\Delta^{\prime} \leq \Delta$.

Proof. Suppose first that $\left(X, L_{0}\right) \in \mathcal{H}_{\Delta}$. According to Corollary $5.2, X$ admits a line bundle $L_{\Delta}$ with $\left(L_{0} \cdot L_{\Delta}\right)=1$ and $\left(L_{\Delta}^{2}\right)=\frac{1-\Delta}{2}=k-4 d^{2}-4$. Choose a symmetric line bundle $L \equiv L_{0}^{2 d} \otimes L_{\Delta}$. Its intersection numbers $\left(L^{2}\right)=4 d^{2}\left(L_{0}^{2}\right)+$ $4 d\left(L_{0} \cdot L_{\Delta}\right)+\left(L_{\Delta}^{2}\right)=4 d(d+1)+k-4$ and $\left(L \cdot L_{0}\right)=2 d\left(L_{0}^{2}\right)+\left(L_{\Delta} \cdot L_{0}\right)=4 d+1$ are positive; hence $L$ is ample. By Lemma 5.3 the line bundle $L_{0}^{2 d} \otimes L_{\Delta}$ is of type $\left(1,2 d(d+1)+\frac{k}{2}-2\right)$. By Proposition 1.1 we may choose $L$ in such a way that $\# X_{2}^{+}(L)=k$. Then by Lemma 1.2, $h^{0}(L)^{-}=\frac{1}{4}\left(\left(L^{2}\right)-\# X_{2}^{+}(L)\right)+2=d(d+1)+1$. So Corollary 3.7 says that there exists an odd divisor $D \in|L|$ with $\operatorname{mult}_{0}(D)=$ 
$2 d+1$, such that $D-\{0\}$ is smooth, and $C=\varphi(D)$ is a rational curve on $K_{X}$. $C$ maps birationally to a rational curve $C^{\prime}=\overline{\pi(C-\{0\})} \subset \mathbb{P}_{2}$ of degree $\operatorname{deg} C^{\prime}=$ $\left(D \cdot L_{0}\right)-\operatorname{mult}_{0}(D)=2 d$ (see Lemma 6.2). Since $D \cap X_{2}=X_{2}^{-}(D)=X_{2}^{+}(L)$, the curve $C^{\prime}$ passes exactly through $k-1$ points of $\left\{l_{i} \cap l_{j}\right\}$, namely the images of $X_{2}^{+}(L)-\{0\}$. Moreover, $C^{\prime}$ is smooth in these points (see Proposition 6.3 (c)), and by Lemma 6.1 $C^{\prime}$ touches the singular lines $l_{i}$ in the remaining intersection points with even multiplicity.

Conversely, suppose the Kummer plane of $\left(X, L_{0}\right)$ admits a curve $C^{\prime}$ as stated above. By Proposition 6.4 there is a line bundle $L \in \mathrm{NS}(X)$ with

$$
\Delta^{\prime}:=\left(L \cdot L_{0}\right)^{2}-2\left(L^{2}\right) \leq 8 d^{2}+9-2 k=\Delta .
$$

This implies the assertion.

As a special case we get Humbert's result for $\Delta=5$, already mentioned in the introduction:

Corollary 7.1 (Humbert). Suppose $\left(X, L_{0}\right)$ is an irreducible principally polarized abelian surface. Then $\left(X, L_{0}\right) \in \mathcal{H}_{5}$ if and only if the associated Kummer plane $\left(\mathbb{P}_{2}, l_{1}, \ldots, l_{6}\right)$ admits a smooth conic passing through five of the 15 points $\left\{l_{i} \cap l_{j}\right\}$ and touching one singular line.

Proof. For the only if implication take Theorem 7.1 with $d=1$ and $k=6$. For the converse implication apply equation (15) in the proof of Proposition 6.4 with $\delta=2$ and $\kappa=5$. This states that $X$ admits a line bundle $L$ with

$$
\Delta:=\left(L_{0} \cdot L\right)^{2}-2\left(L^{2}\right)=5-4 \mu(\mu-1)-8 \nu .
$$

By the Hodge index theorem, $\Delta$ is positive. This implies that $\mu(\mu-1)=0$ and $\nu=0$ and thus $\Delta=5$. This means $\left(X, L_{0}\right) \in \mathcal{H}_{5}$.

Theorem 7.2. Suppose $\Delta=8 d(d+1)+9-2 k$ with integers $d \geq 1$ and $k \in$ $\{4,6,8,10,12\}$. If $\left(X, L_{0}\right) \in \mathcal{H}_{\Delta}$ is an irreducible principally polarized abelian surface, then the associated Kummer plane $\left(\mathbb{P}_{2}, l_{1}, \ldots, l_{6}\right)$ admits a rational curve $C^{\prime}$ of degree $2 d+1$ passing smoothly through exactly $k$ points of $\left\{l_{i} \cap l_{j}\right\}$ and touching the singular lines $l_{i}$ in the remaining intersection points with even multiplicity. Conversely, if $\left(\mathbb{P}_{2}, l_{1}, \ldots, l_{6}\right)$ admits such a curve, then $\left(X, L_{0}\right) \in \mathcal{H}_{\Delta^{\prime}}$ with $\Delta^{\prime} \leq \Delta$.

Proof. Suppose first that $\left(X, L_{0}\right) \in \mathcal{H}_{\Delta}$. According to Corollary $5.2, X$ admits a line bundle $L_{\Delta}$ with $\left(L_{0} \cdot L_{\Delta}\right)=1$ and $\left(L_{\Delta}^{2}\right)=\frac{1-\Delta}{2}=k-4 d(d+1)-4$. Choose a symmetric line bundle $L \equiv L_{0}^{2 d} \otimes L_{\Delta}$. Its intersection numbers $\left(L^{2}\right)=4 d^{2}\left(L_{0}^{2}\right)+$ $4 d\left(L_{0} \cdot L_{\Delta}\right)+\left(L_{\Delta}^{2}\right)=4 d^{2}+k-4$ and $\left(L \cdot L_{0}\right)=2 d\left(L_{0}^{2}\right)+\left(L_{\Delta} \cdot L_{0}\right)=4 d+1$ are positive; hence $L$ is ample. By Lemma 5.3 the line bundle $L_{0}^{2 d} \otimes L_{\Delta}$ is of type $\left(1,2 d^{2}+\frac{k}{2}-2\right)$. By Proposition 1.1 we may choose $L$ in such a way that $\# X_{2}^{-}(L)=k$. Then by Lemma 1.2, $h^{0}(L)^{+}=\frac{1}{4}\left(\left(L^{2}\right)-\# X_{2}^{-}(L)\right)+2=d^{2}+1$. So Corollary 3.7 says that there exists an even divisor $D \in|L|$ with $\operatorname{mult}_{0}(D)=2 d$, such that $D-\{0\}$ is smooth, and $C=\varphi(D)$ is a rational curve on $K_{X}$. $C$ maps birationally to a rational curve $C^{\prime}=\overline{\pi(C-\{0\})} \subset \mathbb{P}_{2}$ of degree $\operatorname{deg} C^{\prime}=\left(D \cdot L_{0}\right)-$ mult $_{0}(D)=2 d+1$ (see Lemma 6.2). Since $D \cap X_{2}-\{0\}=X_{2}^{-}(D)=X_{2}^{-}(L)$, the curve $C^{\prime}$ passes exactly through $k$ points of $\left\{l_{i} \cap l_{j}\right\}$, namely the images of $X_{2}^{-}(L)$. Moreover, $C^{\prime}$ is smooth in these points (see Proposition 6.3(c)), and by Lemma 6.1 $C^{\prime}$ touches the singular lines $l_{i}$ in the remaining intersection points with even multiplicity. 
Conversely, suppose the Kummer plane of $\left(X, L_{0}\right)$ admits a curve $C^{\prime}$ as stated above. By Proposition 6.4 there is a line bundle $L \in \mathrm{NS}(X)$ with

$$
\Delta^{\prime}=\left(L \cdot L_{0}\right)^{2}-2\left(L^{2}\right) \leq 8 d(d+1)+9-2 k=\Delta .
$$

This implies the assertion.

The following two theorems consider the case $\Delta \equiv 0 \bmod 4$. We omit the proofs, since they are completely analogous to those of Theorems 7.1 and 7.2 .

Theorem 7.3. Suppose that $\Delta=8 d^{2}+8-2 k$ with integers $d \geq 1$ and $k \in$ $\{4,6,8,10,12\}$. If $\left(X, L_{0}\right) \in \mathcal{H}_{\Delta}$ is an irreducible principally polarized abelian surface, then the Kummer plane $\left(\mathbb{P}_{2}, l_{1}, \ldots, l_{6}\right)$ of $\left(X, L_{0}\right)$ admits a rational curve $C^{\prime}$ of degree $2 d$ passing smoothly through exactly $k$ points of $\left\{l_{i} \cap l_{j}\right\}$ and touching the singular lines $l_{i}$ in the remaining intersection points with even multiplicity. Conversely, if $\left(\mathbb{P}_{2}, l_{1}, \ldots, l_{6}\right)$ admits such a curve, then $\left(X, L_{0}\right) \in \mathcal{H}_{\Delta^{\prime}}$ with $\Delta^{\prime} \leq \Delta$.

Theorem 7.4. Suppose $\Delta=8 d(d+1)+12-2 k$ with integers $d \geq 1$ and $k \in$ $\{4,6,8,10,12\}$. If $\left(X, L_{0}\right) \in \mathcal{H}_{\Delta}$ is an irreducible principally polarized abelian surface, then the associated Kummer plane $\left(\mathbb{P}_{2}, l_{1}, \ldots, l_{6}\right)$ admits a rational curve $C^{\prime}$ of degree $2 d+1$ passing smoothly through exactly $k-1$ points of $\left\{l_{i} \cap l_{j}\right\}$ and touching the singular lines $l_{i}$ in the remaining intersection points with even multiplicity. Conversely, if $\left(\mathbb{P}_{2}, l_{1}, \ldots, l_{6}\right)$ admits such a curve, then $\left(X, L_{0}\right) \in \mathcal{H}_{\Delta^{\prime}}$ with $\Delta^{\prime} \leq \Delta$.

The following two theorems are due to Humbert.

Theorem 7.5. If $\left(X, L_{0}\right) \in \mathcal{H}_{\delta^{2}}$ is an irreducible principally polarized abelian surface, then the Kummer plane $\left(\mathbb{P}_{2}, l_{1}, \ldots, l_{6}\right)$ of $\left(X, L_{0}\right)$ admits an irreducible rational curve $C^{\prime}$ of degree $\delta-1$ passing smoothly through exactly three points of $\left\{l_{i} \cap l_{j}\right\}$ and touching the singular lines $l_{i}$ in the remaining intersection points with even multiplicity. Conversely, if $\left(\mathbb{P}_{2}, l_{1}, \ldots, l_{6}\right)$ admits such a curve, then $\left(X, L_{0}\right) \in \mathcal{H}_{\Delta^{\prime}}$ with

$$
\Delta^{\prime} \leq \begin{cases}2 \delta^{2}-4 \delta+4 & \text { if } \delta \text { is even }, \\ 2 \delta^{2}-4 \delta+3 & \text { if } \delta \text { is odd }\end{cases}
$$

Proof. We give the proof in the case $\delta=2 d$. The proof of the odd case is analogous. Suppose first that $\left(X, L_{0}\right) \in \mathcal{H}_{\delta^{2}}=\mathcal{H}_{4 d^{2}}$. According to Corollary [5.2 $X$ admits a line bundle $L_{\Delta}$ with $\left(L_{0} \cdot L_{\Delta}\right)=0$ and $\left(L_{\Delta}^{2}\right)=-\frac{\Delta}{2}=-2 d^{2}$. Choose a symmetric line bundle $L \equiv L_{0}^{d} \otimes L_{\Delta}$. Its intersection numbers are $\left(L^{2}\right)=d^{2}\left(L_{0}^{2}\right)+\left(L_{\Delta}^{2}\right)=$ $2 d^{2}-2 d^{2}=0$ and $\left(L \cdot L_{0}\right)=d\left(L_{0}^{2}\right)+\left(L_{\Delta} \cdot L_{0}\right)=2 d$. Hence there exists an elliptic curve $E$ on $X$ such that $L=\mathcal{O}_{X}(E)$. One may choose $L$ or $E$ respectively in such a way that $E$ is a subgroup of $X$. In particular, $E$ passes through four 2-torsion points including 0. By Hurwitz's Theorem the image $C=\varphi(E)$ is a smooth rational curve of degree $\operatorname{deg} C=\left(E \cdot L_{0}\right)=2 d=\delta$ on $K_{X}$. $C$ maps birationally to a rational curve $C^{\prime}=\overline{\pi(C-\{0\})} \subset \mathbb{P}_{2}$ of degree $\operatorname{deg} C^{\prime}=\left(E \cdot L_{0}\right)-$ mult $_{0}(E)=\delta-1$ (see Lemma 6.2). The curve $C^{\prime}$ passes exactly through 3 points of $\left\{l_{i} \cap l_{j}\right\}$, namely the images of $E \cap X_{2}-\{0\}$. Moreover, $C^{\prime}$ is smooth in these points (see Proposition6.3 (c)), and by Lemma 6.1, $C^{\prime}$ touches the singular lines $l_{i}$ in the remaining intersection points with even multiplicity.

Conversely, suppose the Kummer plane of $\left(X, L_{0}\right)$ admits a curve $C^{\prime}$ as stated above. By Proposition 6.4 there is a line bundle $L \in \mathrm{NS}(X)$ with

$$
\Delta^{\prime}=\left(L \cdot L_{0}\right)^{2}-2\left(L^{2}\right) \leq 8 d^{2}-8 d+4=2 \delta^{2}-4 \delta+4 .
$$


This implies the assertion.

The next theorem presents the characterizations by curves of genus $\geq 1$.

Theorem 7.6. Suppose $d$ and $g$ are integers $\geq 1$.

(1) If $\left(X, L_{0}\right) \in \mathcal{H}_{8\left(d^{2}-g\right)}$ is an irreducible principally polarized abelian surface, then the associated Kummer plane $\left(\mathbb{P}_{2}, l_{1}, \ldots, l_{6}\right)$ admits a g-dimensional linear system of curves $C^{\prime}$ of genus $g$ and degree $2 d$ all of which pass through the four points $l_{1} \cap l_{2}, l_{2} \cap l_{3}, l_{3} \cap l_{4}, l_{4} \cap l_{1}$ and touch the singular lines $l_{i}$ in the remaining intersection points with even multiplicity. Conversely, if $\left(\mathbb{P}_{2}, l_{1}, \ldots, l_{6}\right)$ admits such a linear system, then $\left(X, L_{0}\right) \in \mathcal{H}_{\Delta}$ with $\Delta \leq$ $8\left(d^{2}-g\right)$.

(2) If $\left(X, L_{0}\right) \in \mathcal{H}_{8(d(d+1)-g)+4}$ is an irreducible principally polarized abelian surface, then the associated Kummer plane $\left(\mathbb{P}_{2}, l_{1}, \ldots, l_{6}\right)$ admits a g-dimensional linear system of curves $C^{\prime}$ of genus $g$ and degree $2 d+1$ all of which pass through six of the points $\left\{l_{i} \cap l_{j}\right\}$ and touch the singular lines $l_{i}$ in the remaining intersection points with even multiplicity. Conversely, if $\left(\mathbb{P}_{2}, l_{1}, \ldots, l_{6}\right)$ admits such a linear system, then $\left(X, L_{0}\right) \in \mathcal{H}_{\Delta}$ with $\Delta \leq 8(d(d+1)-g)+4$.

(3) If $\left(X, L_{0}\right) \in \mathcal{H}_{8\left(d^{2}-g\right)+1}$ is an irreducible principally polarized abelian surface, then the associated Kummer plane $\left(\mathbb{P}_{2}, l_{1}, \ldots, l_{6}\right)$ admits a g-dimensional linear system of curves $C^{\prime}$ of genus $g$ and degree $2 d$ all of which pass through the three points $l_{1} \cap l_{2}, l_{2} \cap l_{3}, l_{3} \cap l_{1}$ and touch the singular lines $l_{i}$ in the remaining intersection points with even multiplicity. Conversely, if $\left(\mathbb{P}_{2}, l_{1}, \ldots, l_{6}\right)$ admits such a linear system, then $\left(X, L_{0}\right) \in \mathcal{H}_{\Delta}$ with $\Delta \leq 8\left(d^{2}-g\right)+1$.

(4) If $\left(X, L_{0}\right) \in \mathcal{H}_{8\left(d^{2}-g\right)-3}$ is an irreducible principally polarized abelian surface, then the associated Kummer plane $\left(\mathbb{P}_{2}, l_{1}, \ldots, l_{6}\right)$ admits a g-dimensional linear system of curves $C^{\prime}$ of genus $g$ and degree $2 d$ all of which pass through the five points $l_{1} \cap l_{2}, l_{2} \cap l_{3}, l_{3} \cap l_{4}, l_{4} \cap l_{5}, l_{5} \cap l_{1}$ and touch the singular lines $l_{i}$ in the remaining intersection points with even multiplicity. Conversely, if $\left(\mathbb{P}_{2}, l_{1}, \ldots, l_{6}\right)$ admits such a linear system, then $\left(X, L_{0}\right) \in \mathcal{H}_{\Delta}$ with $\Delta \leq 8\left(d^{2}-g\right)-3$.

Here in each case the six lines are meant to be numbered suitably.

Proof. (1) Suppose first that $\left(X, L_{0}\right) \in \mathcal{H}_{8\left(d^{2}-g\right)}$. According to Corollary [5.2, $X$ admits a line bundle $L_{\Delta}$ with $\left(L_{0} \cdot L_{\Delta}\right)=0$ and $\left(L_{\Delta}^{2}\right)=-\frac{\Delta}{2}=4\left(g-d^{2}\right)$. Choose a symmetric line bundle $L \equiv L_{0}^{2 d} \otimes L_{\Delta}$. Its intersection numbers $\left(L^{2}\right)=4 d^{2}\left(L_{0}^{2}\right)+$ $\left(L_{\Delta}^{2}\right)=4\left(d^{2}+g\right)$ and $\left(L \cdot L_{0}\right)=2 d\left(L_{0}^{2}\right)=4 d$ are positive; hence $L$ is ample. By Lemma 5.3 the line bundle $L_{0}^{2 d} \otimes L_{\Delta}$ is of type $\left(1,2\left(d^{2}+g\right)\right)$. By Proposition 1.1 we may choose $L$ in such a way that $\# X_{2}^{-}(L)=4$. Then by Lemma 1.2 $h^{0}(L)^{+}=\frac{1}{4}\left(\left(L^{2}\right)-\# X_{2}^{-}(L)\right)+2=d^{2}+g+1$. So Corollary 3.7 says that the general member $D$ of the linear system $\left|L \otimes \mathcal{I}_{0}^{2 d}\right|^{+}$is smooth on $X-\{0\}$, and maps to curve $C=\varphi(D)$ of geometric genus $g_{C}=g$ on $K_{X}$. C maps birationally to a curve $C^{\prime}=\overline{\pi(C-\{0\})} \subset \mathbb{P}_{2}$ of degree $\operatorname{deg} C^{\prime}=\left(D \cdot L_{0}\right)-$ mult $_{0}(D)=4 d-2 d=2 d$ (see Lemma 6.2). Since $D \cap X_{2}=X_{2}^{-}(D)=X_{2}^{-}(L)$, the curve $C^{\prime}$ passes exactly through 4 points of $\left\{l_{i} \cap l_{j}\right\}$, namely the images of $X_{2}^{-}(L)$ and by Lemma 6.1, $C^{\prime}$ touches the singular lines $l_{i}$ in the remaining intersection points with even multiplicity. Because of this the 4 points in $\left\{l_{i} \cap l_{j}\right\}$ must be of the form $l_{1} \cap l_{2}, l_{2} \cap l_{3}, l_{3} \cap l_{4}, l_{4} \cap l_{1}$. 
Conversely, suppose the Kummer plane of $\left(X, L_{0}\right)$ admits a linear system as stated above. Take a general member $C^{\prime}$ of this linear system. By Proposition 6.4 there is a line bundle $L \in \mathrm{NS}(X)$ with

$$
\Delta:=\left(L \cdot L_{0}\right)^{2}-2\left(L^{2}\right) \leq 8 d^{2}-8-8 g+8=8\left(d^{2}-g\right) .
$$

This implies assertion (1). The proofs of (2), (3), and (4) follow the same pattern.

As we saw in the proof of Corollary 17.1, applying equation (15) rather than Proposition 6.4 yields a more precise result for small $\Delta$. Similarly, Humbert's results for $\Delta=4,8,9$, and 12 are consequences of the Theorems above.

Corollary 7.2. Suppose $\left(X, L_{0}\right)$ is an irreducible principally polarized abelian surface.

$\Delta=4:\left(X, L_{0}\right) \in \mathcal{H}_{4}$ if and only if (numbering the 6 lines on its Kummer plane $\left(\mathbb{P}_{2}, l_{1}, \ldots, l_{6}\right)$ suitably) the three points $l_{1} \cap l_{2}, l_{3} \cap l_{4}, l_{5} \cap l_{6}$ are collinear.

$\Delta=8:$ If $\left(X, L_{0}\right) \in \mathcal{H}_{8}$, then the associated Kummer plane $\left(\mathbb{P}_{2}, l_{1}, \ldots, l_{6}\right)$ admits a smooth conic passing through four of the 15 points $\left\{l_{i} \cap l_{j}\right\}$ and touching the singular lines $l_{i}$ in the remaining intersection points with even multiplicity. Conversely, if $\left(\mathbb{P}_{2}, l_{1}, \ldots, l_{6}\right)$ admits such a curve, then $\left(X, L_{0}\right) \in \mathcal{H}_{\Delta}$ with $\Delta=8$ or 4 .

$\Delta=9:\left(X, L_{0}\right) \in \mathcal{H}_{9}$ if and only if its Kummer plane $\left(\mathbb{P}_{2}, l_{1}, \ldots, l_{6}\right)$ admits a smooth conic passing through three of the 15 points $\left\{l_{i} \cap l_{j}\right\}$ and touching the singular lines $l_{i}$ in the remaining intersection points with even multiplicity.

$\Delta=12$ : If $\left(X, L_{0}\right) \in \mathcal{H}_{12}$, then its Kummer plane $\left(\mathbb{P}_{2}, l_{1}, \ldots, l_{6}\right)$ admits a cubic passing smoothly through three of the 15 points $\left\{l_{i} \cap l_{j}\right\}$ and touching the singular lines $l_{i}$ in the remaining intersection points with even multiplicity. Conversely, if $\left(\mathbb{P}_{2}, l_{1}, \ldots, l_{6}\right)$ admits such a curve, then $\left(X, L_{0}\right) \in \mathcal{H}_{\Delta}$ with $\Delta=12$ or 8 .

Proof. For the case $\Delta=4$ apply Theorem 7.5 for $\Delta=8$ apply Theorem 7.3 with $d=1$ and $k=4$, for $\Delta=9$ apply Theorem 7.1 with $d=1$ and $k=4$, and for $\Delta=12$ apply Theorem 7.4 with $d=1$ and $k=8$.

In the cases $\Delta=13,16,17,20$ and 21 we obtain similarly

Corollary 7.3. Suppose $\left(X, L_{0}\right)$ is an irreducible principally polarized abelian surface.

$\Delta=13:$ If $\left(X, L_{0}\right) \in \mathcal{H}_{13}$, then its Kummer plane $\left(\mathbb{P}_{2}, l_{1}, \ldots, l_{6}\right)$ admits a cubic passing smoothly through six of the 15 points $\left\{l_{i} \cap l_{j}\right\}$ and touching the singular lines $l_{i}$ in the remaining intersection points with even multiplicity. Conversely, if $\left(\mathbb{P}_{2}, l_{1}, \ldots, l_{6}\right)$ admits such a curve, then $\left(X, L_{0}\right) \in \mathcal{H}_{\Delta}$ with $\Delta=13$ or 5 .

$\Delta=16:$ If $\left(X, L_{0}\right) \in \mathcal{H}_{16}$, then its Kummer plane $\left(\mathbb{P}_{2}, l_{1}, \ldots, l_{6}\right)$ admits a cubic passing smoothly through three of the 15 points $\left\{l_{i} \cap l_{j}\right\}$ and touching the singular lines $l_{i}$ in the remaining intersection points with even multiplicity. Conversely, if $\left(\mathbb{P}_{2}, l_{1}, \ldots, l_{6}\right)$ admits such a curve, then $\left(X, L_{0}\right) \in \mathcal{H}_{\Delta}$ with $\Delta \in\{4,8,12,16,20\}$.

$\Delta=17:$ If $\left(X, L_{0}\right) \in \mathcal{H}_{17}$, then its Kummer plane $\left(\mathbb{P}_{2}, l_{1}, \ldots, l_{6}\right)$ admits a cubic passing smoothly through three of the 15 points $\left\{l_{i} \cap l_{j}\right\}$ and touching the 
singular lines $l_{i}$ in the remaining intersection points with even multiplicity. Conversely, if $\left(\mathbb{P}_{2}, l_{1}, \ldots, l_{6}\right)$ admits such a curve, then $\left(X, L_{0}\right) \in \mathcal{H}_{\Delta}$ with $\Delta \in\{1,9,17\}$.

$\Delta=20:$ If $\left(X, L_{0}\right) \in \mathcal{H}_{20}$, then its Kummer plane $\left(\mathbb{P}_{2}, l_{1}, \ldots, l_{6}\right)$ admits a cubic passing smoothly through three of the 15 points $\left\{l_{i} \cap l_{j}\right\}$ and touching the singular lines $l_{i}$ in the remaining intersection points with even multiplicity. Conversely, if $\left(\mathbb{P}_{2}, l_{1}, \ldots, l_{6}\right)$ admits such a curve, then $\left(X, L_{0}\right) \in \mathcal{H}_{\Delta}$ with $\Delta \in\{4,8,12,16,20\}$.

$\Delta=21$ : If $\left(X, L_{0}\right) \in \mathcal{H}_{21}$, then its Kummer plane $\left(\mathbb{P}_{2}, l_{1}, \ldots, l_{6}\right)$ admits a curve of degree four passing smoothly through nine of the 15 points $\left\{l_{i} \cap l_{j}\right\}$ and touching the singular lines $l_{i}$ in the remaining intersection points with even multiplicity. Conversely, if $\left(\mathbb{P}_{2}, l_{1}, \ldots, l_{6}\right)$ admits such a curve, then $\left(X, L_{0}\right) \in \mathcal{H}_{\Delta}$ with $\Delta \in\{5,13,21\}$.

\section{REFERENCES}

[ACGH] Arbarello, E., Cornalba, M., Griffiths, P. A., and Harris, J., Geometry of Algebraic Curves, Volume I, Grundlehren der Mathematischen Wissenschaften, Springer-Verlag, Berlin, 1985. MR 86h:14019

[B] Bauer, Th., Projective images of Kummer surfaces, Math. Ann. 299,155-170 (1994) MR 95c: 14040

[CAV] Lange, H. and Birkenhake, Ch., Complex Abelian Varieties, Grundlehren der Mathematischen Wissenschaften, Springer-Verlag, Berlin, 1992. MR 94j:14001

[CT] Birkenhake, Ch. and Lange, H., Complex Tori, Progress in Math., Bd. 177, Birkhäuser (1999) MR 2001k:14085

[GD] Gonzalez-Dorrego, M. R., $(16,6)$ Configurations and Geometry of Kummer Surfaces in $\mathbb{P}^{3}$. Memoirs of Amer. Math. Soc., Vol. 107 (1994). MR 94f:14034

[GH] Griffiths, P. A. and Harris, J., Principles of Algebraic Geometry, John Wiley \& Sons, New York (1978) MR 80b:14001

[H] Humbert, G., Sur les fonctionnes abéliennes singulières. I, II, III. J. Math. Pures Appl. serie 5, t. V, 233-350 (1899); t. VI, 279-386 (1900); t. VII, 97-123 (1901)

[vdG] van der Geer, G., Hilbert modular surfaces, Ergebnisse der Mathematik und ihrer Grenzgebiete, Springer-Verlag, Berlin, 1988. MR 89c:11073

[W] Wilhelm, H., Geometrische Charakterisierung von Kummerflächen, Dissertation, Erlangen (1998)

Christina Birkenhake, Universität Mainz, Staudingerweg 9, D-55099 Mainz, Germany

E-mail address: birken@Mathematik.uni-mainz.de

Hannes Wilhelm, 10 Studley Count, 4 Jamestown Way, London E14 2DA, England

E-mail address: Hannes.Wilhelm@dresdner-bank.com 